\title{
Targeted Sound Attenuation Capacity of 3D Printed Noise Cancelling Waveguides
}

\author{
Arun Arjunan ${ }^{\mathrm{a} *}$ \\ a Faculty of Science and Engineering, University of Wolverhampton, Telford Campus, \\ Priorslee TF2 9NT, UK
}

\begin{abstract}
The study explores the creation of 3D printed sound cancelling waveguides that can be customised for selected frequencies as a function of geometry. The potential for attenuation in these waveguides is characterised through experimentally measured acoustic-absorption $(\alpha)$ and Transmission Loss (TL). This was done to evaluate the potential of geometry-controlled waveguides in the development of passive sound cancelling structures. Geometrically complex waveguides to exploit the Herschel-Quincke-Arjunan (HQA) waveguide model manufactured in Nylon-12 using Selective Laser Melting (SLM) are presented. The attenuation of the waveguides was compared to the bulk Nylon-12 materials to segregate the material-based influence. The results showed that the performance of HQA waveguides can be controlled as a function of length, diameter and waveguide-tortuosity. Accordingly, under right parameters significant improvement in $\alpha(0.96,0.80,0.61$ and 0.98$)$ and TL (65.59\%, $30.15 \%, 53.36 \%$ and $95.28 \%$ ) can be achieved at the design frequency. The proposed methodology can be used to develop customisable waveguides exploiting the principles of acoustic wave interference for a range of application including building walls, noise barriers and absorptive panels.
\end{abstract}

Keywords: 3D Printing; Nylon-12, Transmission Loss; Sound Cancelling; Computer Aided Design; Impedance Tube.

\footnotetext{
* Author:

Tel.: +44 1902 323829; fax: +4401902323843

E-mail address: a.arjunan@wlv.ac.uk (Dr. Arun Arjunan)
} 


\section{Introduction}

For buildings close to polluted noise environments such as motorways, railways and airports, noise reduction is still a challenging problem [1]. Due to high population densities, the effect of noise pollution is a dominant issue in Europe. more than half a million A significant number of people ( $<0.5$ million) move home every year in England as a result of noise pollution [2]. This is despite the adoption of numerous approaches taken by United Kingdom to manage the impact of sound-pollution to resident communities. Solving this challenging problem demands new and innovative design-based solutions that can provide targeted noise reduction.

Building components that act as noise defenders have their performance often dictated by its mass and stiffness properties [3-7]. Accordingly, these components require significant addition in mass and thickness to make meaningful improvements to acoustic insulation. Furthermore, the traditional techniques of mass and stiffness dampening do not allow for frequency customisation. No frequency customisation means that key noise sources (traffic noise, railway noise, airport noise etc.) cannot be targeted leading to poor efficiency.

Frequency dependent noise reduction techniques are of significant interest to the building community. Targeting the frequency spectrum of the noise source allows to design acoustically fit for purpose building components leading to stress friendly infrastructure. This will also enable the targeted reduction of low frequency or high frequency noise which are often difficult to manage through traditional methodologies $[8,9]$.

Active noise cancellation using sound-interference has long been identified as a potential technology for targeted noise reduction [10-15]; common example being noise cancelling headphones [16]. However, this is often implemented in an active form, meaning it require additional energy and employs secondary acoustic source [17-19]. On the other hand, studies on the implementation of geometry or structure-based resonance without the use of active elements has been gaining momentum [20-24].

Sound transmissions in two-dimensional tubes were explored by Depollier et al. [25] and demonstrated attenuation potential. Later on, a general expression for the acoustic insulation of connected waveguides under one dimensional flow utilising the principle of interference was proposed by Selamet [26]. This work was based on the Herschel-Quincke (HQ) theory of interference-based noise reduction [27]. However, the focus was on a one-dimensional lined duct and involved a two-tube concept. Despite the available literature on resonance based waveguides, research focusing on application to building systems are limited [13,22].

The attenuation potential of interference channels with a view to applying it to building components was carried out through the investigation of curved waveguides and coplanar voids 
[28-30]. Where these pioneering works established the attenuation potential that can be achieved, none of these works characterised the transmission loss associated. Other notable works building on geometrical parameters combined the principles of Helmholtz resonators with flexible tubes and neck extensions by Shinsuke [31] and $\mathrm{Lu}$ [32]. Attenuation of high amplitude pulses using a lattice of Helmholtz resonators was studied by Olivier [33] and reported a nonlinear absorption. Recent analysis on quarter wavelength resonators were carried out by Cambonie et al. [34] who studied the effect of curvature while the effect of orifice geometries were quantified by Howard and Craig [35].

The rise of digital manufacturing techniques such as Selective Laser Melting (SLM) [36] allows the creation of complex shaped waveguides. Consequently, SLM is increasing being used in the development of acoustic materials due to the ease at which geometry can be customised $[37,38]$. Zhang et al. [39] developed continuously graded phononic crystals using SLS that showed broadband noise reduction potential between 1.350 and $3 \mathrm{kHz}$. Acoustic properties of a 3D printed material with micro-tubes manufactured using SLS was studied by Chaoyang et al. [40] which also showed elevated acoustic properties.

As can be seen there is a substantial focus from the research community towards design-based solutions to improve acoustic absorption. However, despite the existing literature on absorption, TL performance associated with passive interference waveguides are limited. From a practical standpoint, a combination of these two characteristics are of significance to fully realise designbased solutions for noise reduction. Accordingly, this study explores the potential of developing interference waveguides that can have a potential impact on both the sound absorption coefficient as well as transmission loss. A non-traditional design approach integrating geometrical parameters into a single cavity dictated by the incident acoustic frequency is demonstrated. This approach was realised through taking advantage of the SLM manufacturing process in Nylon-12 material. Emphasis was placed on cavity design strategy at four selected frequencies between 0.4 and $1.6 \mathrm{kHz}$. A geometrical parametric analysis is also carried to understand sound-interference in open channel waveguides in addition to extending the theory to propose the HerschelQuincke-Arjunan (HQA) model of branchless sound-interference waveguides. Therefore, this research establishes a new direction in the area of design-based performance of both $\alpha$ and TL suitable for wider acoustic application.

\section{Methodology}

\subsection{Herschel-Quincke-Arjunan (HQA) waveguide for interference}

As shown in Fig. 1; interference of sound waves happens when two sound waves W1 and W2 overlapped to form a resultant wave I. The resultant wave has a higher or lower amplitude depends on Constructive-Interference (CI) or Destructive-Interference (DI) respectively. The amount of interference depends on the acoustic phase shift between the waves. The physics of 
sound-interference is well known and is widely exploited in active form referred to as Active Sound Cancellation (ASC). The most common application of ASC can be found in noise cancelling headphones and similar controlled acoustic environments [41]. ASC require a sound monitoring device such as a microphone that inputs the acoustic wave into an electronic circuit where a phase shifted wave at $180^{\circ}$ is produced which is then added to the incoming sound wave to cause CI or DI. However, this study attempts to create phase shift to an acoustic wave to create DI through geometry-based intervention alone without using any active components. Attempts in this regard have been scarce and focused on multi-channel waveguides with varying degree of acoustic performances [28-30].

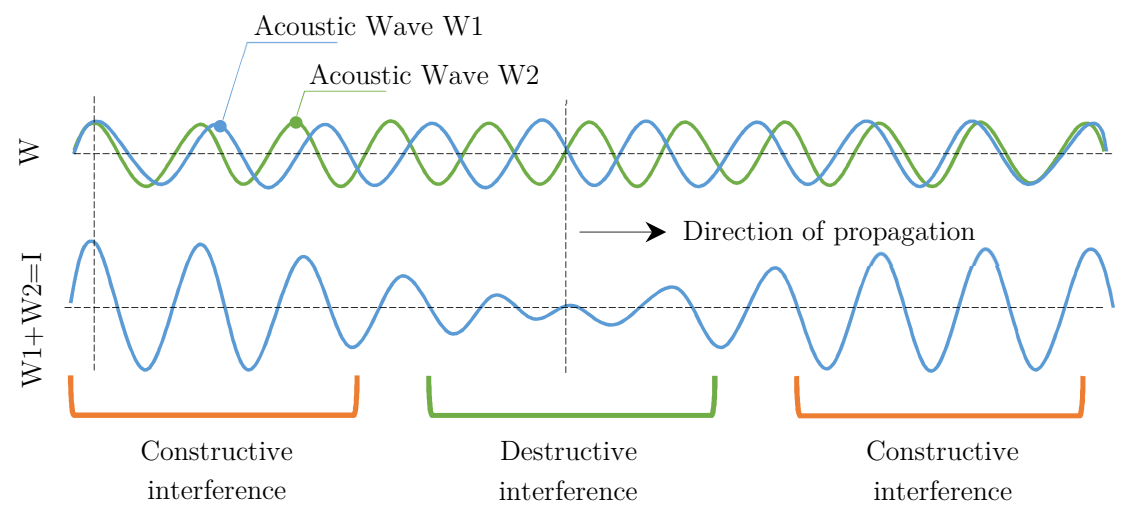

Fig. 1. Sound-interference through superimposition of sound waves W1 and W2.

For geometry-based creation of DI, a Herschel-Quincke-Arjunan (HQA) single channel waveguide is proposed as shown in Fig. 2. When acoustic waves simultaneously enter through both ends of the HQA waveguide of distance $l$, they are hypothesised to meet at a certain phase difference to create DI. The degree of phase shift $(\varphi)$ between the acoustic waves can be controlled by modifying the waveguide length $(l)$ and the wavelength $(\lambda)$ of the sound waves. When the difference in wavelength is half the incident wavelength, $\varphi$ will become $\pi$ radians $\left(180^{\circ}\right)$ and perfect DI will occur leading to complete passive noise cancellation.

Considering the HQA waveguide in Fig. 2; acoustic waves from the same source simultaneously enter the waveguide of total length $l$ under a constant speed of sound $c$. The frequency of sound and the sound transmission-length-difference $\Delta l$ can then be related using Eqn. (1):

$$
\Delta l=l_{2}-l_{1}=\frac{(2 m-1) c}{2 f}
$$

Where $m$ represents the occurrence of DI starting at 1 tending to infinity $(\infty)$ in a positive integral manner $(m=1,2,3 \ldots \ldots \ldots \infty)$ and $\Delta l$ refers to the point where DI will occur which is the difference between the long $\left(l_{1}\right)$ and short $\left(l_{2}\right)$ path of the HQA waveguide as shown in Fig. 2 . 


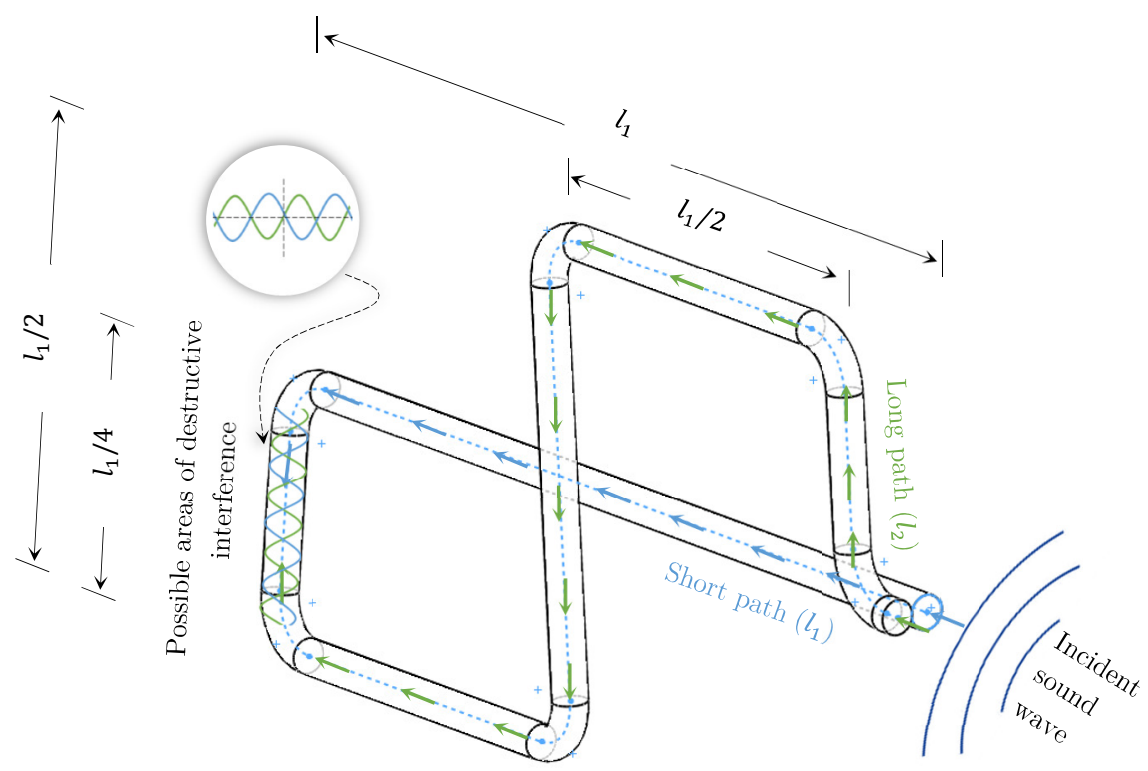

Fig. 2. Herschel-Quincke-Arjunan (HQA) single channel waveguide to create geometry-based destructive interference.

The HQA concept builds on the one-dimensional (1D) theory [26] of the HQ tube shown in Fig. 3. The HQ tube is composed of a straight and branched channel where the ends are connected to the sides of the main duct experiencing a unidirectional flow. This concept was first introduced by Herschel [42] and verified by Quincke cited in [43] using the HQ tube experiment under a unidirectional flow. The HQ tube works on the principle that a particular frequency of acoustic wave divided through two channels, one being longer than $0.5 \lambda$, then the waves will experience a certain phase difference.

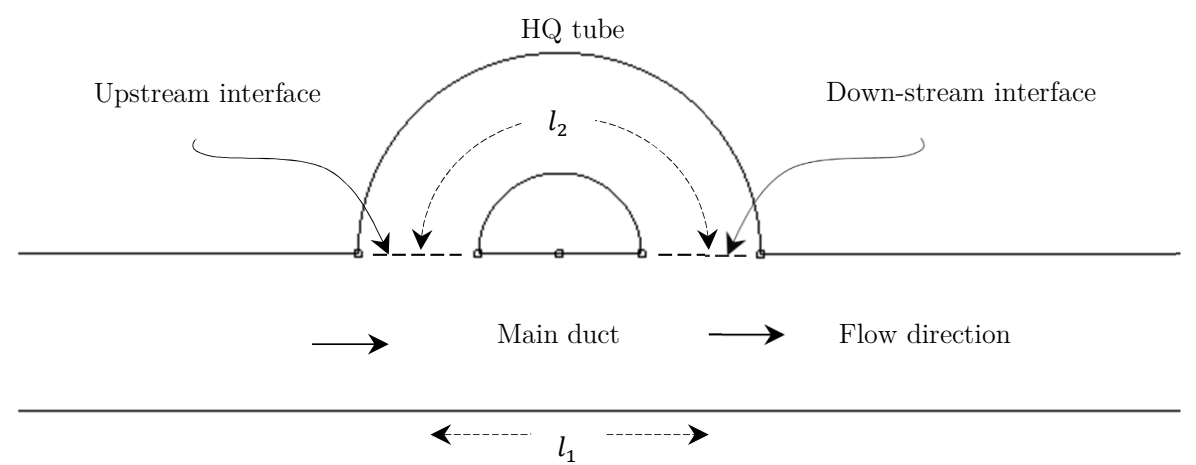

Fig. 3. The Herschel-Quincke (HQ) tube.

Even though, the HQ tube has specialist application for duct acoustics in the presence of unidirectional flow, research is necessary to identify alternative interference waveguides to deliver noise cancellation for general application such as building walls or noise barriers. Accordingly, the fundamental aim of this study was to validate the HQA hypothesis and to characterise the DI that can be achieved. Efforts have been placed to implement the proposed theory into 
geometries so that DI can occur at the external surface (mouth) of the waveguide. This will enable HQA waveguides to be used as passive sound cancellation devices. This means that geometry-based DI can be exploited for targeted $\alpha$ or TL establishing a new relationship in the field of geometrically controlled acoustic performance.

\subsection{Design generation}

The derivation of the HQA waveguide for experimental samples are conducted following Eqn. (1), where the speed of sound is taken as $343.0 \mathrm{~m} / \mathrm{s}$ and ratio of waveguide length $(l)$ to transmission-length-difference $(\Delta l)$ is equated using Eqn. (2):

$$
\frac{l}{\Delta l}=1.0
$$

For HQA waveguides satisfying Eqn. (2), noise cancellation is anticipated at the surface of the waveguide. Accordingly, the lengths of the waveguides are calculated to be $34.3 \mathrm{~cm}$ (A), 17.15 cm (B) and $11.43 \mathrm{~cm}$ (C) for noise cancellation to occur at 500, 1000 and $1500 \mathrm{~Hz}$ respectively. In all design cases a minimum requirement of $1 \mathrm{~cm}$ wall thickness was considered to minimise in direct transmission through the boundaries of the waveguide.

For each transmission-length-difference $(\Delta l)$, four waveguides are designed varying the diameter as shown in Table 1 . The upper limit of the diameter $(3 \mathrm{~cm})$ was the largest that could hold the longest $\Delta l$ within the specimen dimension limited by the impedance tube diameter to conduct the low frequency analysing (400 to $1600 \mathrm{~Hz}$ ).

Table 1 Parametric values considered for the experimental samples (all dimensions in $\mathrm{cm}$ ).

\begin{tabular}{|c|c|c|c|c|c|c|c|c|}
\hline Type & Waveguide $(\Delta l)$ & $d 1$ & $d 2$ & $d 3$ & $d 4$ & $\begin{array}{c}\text { Min wall } \\
\text { thickness } \\
(t)\end{array}$ & $\begin{array}{l}\text { Specimen } \\
\text { height }(\boldsymbol{h})\end{array}$ & $\begin{array}{c}\text { Specimen } \\
\text { diameter }(D)\end{array}$ \\
\hline $\mathrm{U}$ & $34.3(\mathrm{~A})$ & $\begin{array}{c}3 \\
(3 \mathrm{AU})\end{array}$ & $\begin{array}{c}2.5 \\
(2.5 \mathrm{AU})\end{array}$ & $\begin{array}{c}2 \\
(2 \mathrm{AU})\end{array}$ & $\begin{array}{c}1.5 \\
(1.5 \mathrm{AU})\end{array}$ & 1.0 & 10 & 10 \\
\hline $\mathrm{U}$ & $17.15(\mathrm{~B})$ & $\begin{array}{c}3 \\
(3 \mathrm{BU})\end{array}$ & $\begin{array}{c}2.5 \\
(2.5 \mathrm{BU})\end{array}$ & $\begin{array}{c}2 \\
(2 \mathrm{BU})\end{array}$ & $\begin{array}{c}1.5 \\
(1.5 \mathrm{BU})\end{array}$ & 1.0 & 10 & 10 \\
\hline $\mathrm{U}$ & $11.43(\mathrm{C})$ & $\begin{array}{c}3 \\
(3 \mathrm{CU})\end{array}$ & $\begin{array}{c}2.5 \\
(2.5 \mathrm{CU})\end{array}$ & $\begin{array}{c}2 \\
(2 \mathrm{CU})\end{array}$ & $\begin{array}{c}1.5 \\
(1.5 \mathrm{CU})\end{array}$ & 1.0 & 10 & 10 \\
\hline $\mathrm{H}$ & $34.3(\mathrm{~A})$ & - & - & $\begin{array}{c}2 \\
(2 \mathrm{AH})\end{array}$ & - & 1.0 & 10 & 10 \\
\hline I & $34.3(\mathrm{~A})$ & - & - & $\begin{array}{c}2 \\
(2 \mathrm{AI})\end{array}$ & - & 1.0 & 10 & 10 \\
\hline $\mathrm{Q}$ & $8.58(\mathrm{D})$ & - & - & - & $\begin{array}{c}1.5 \\
(1.5 \mathrm{DQ})\end{array}$ & 1.0 & 10 & 10 \\
\hline $\mathrm{S}$ & Solid specimen $(\mathrm{N})$ & $\begin{array}{c}0 \\
(\mathrm{ONS})\end{array}$ & - & - & - & - & 10 & 10 \\
\hline
\end{tabular}



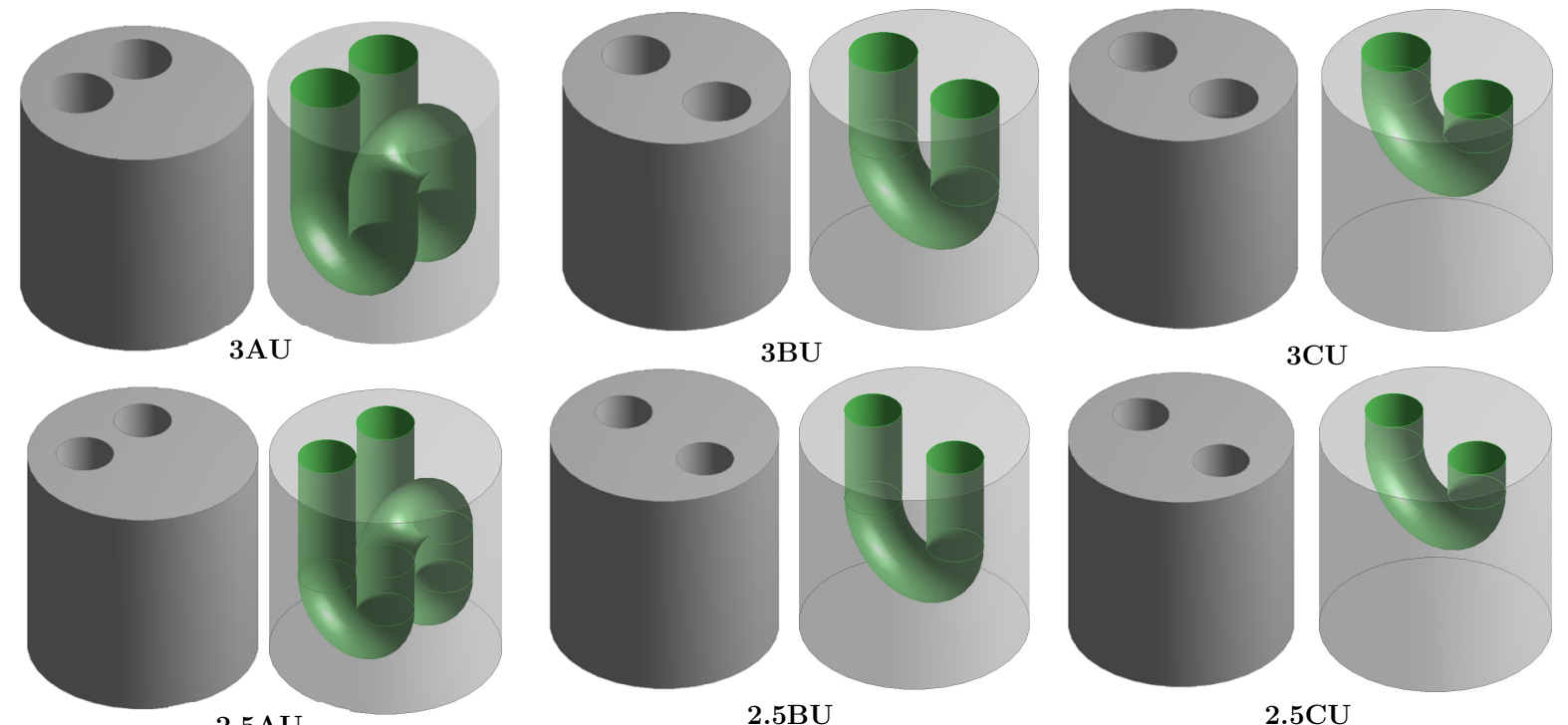

$2.5 \mathrm{BU}$

$2.5 \mathrm{CU}$
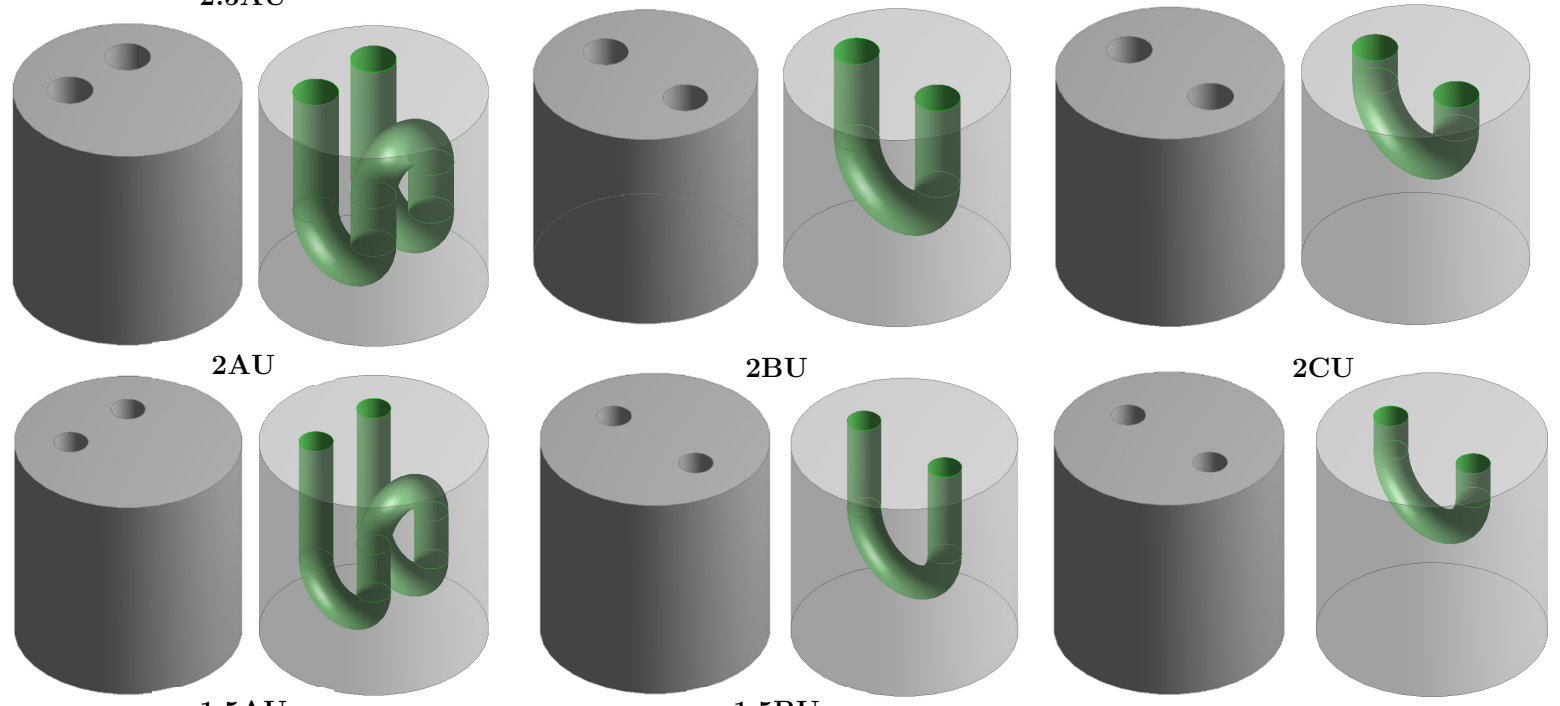

$1.5 \mathrm{AU}$

$1.5 \mathrm{BU}$
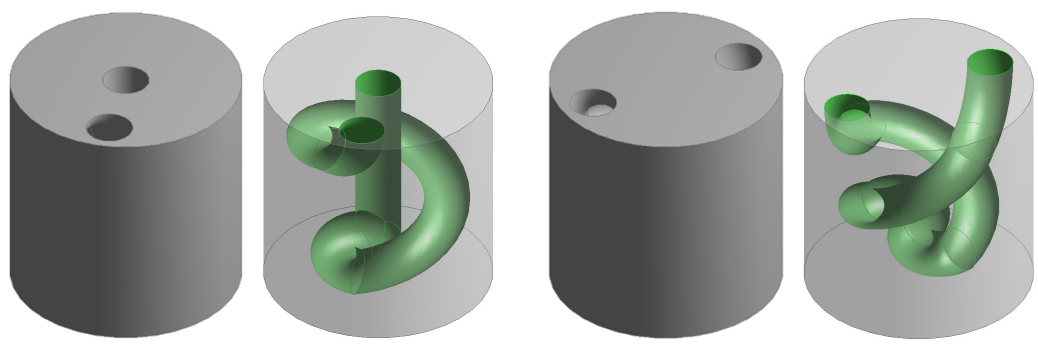

2AI

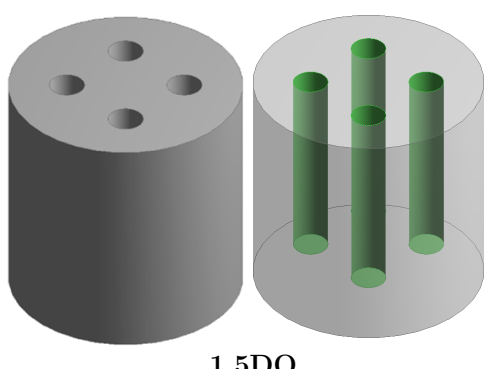

$2 \mathrm{AH}$

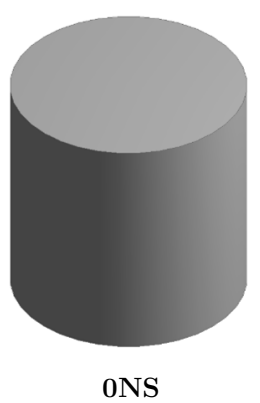

$1.5 \mathrm{DQ}$
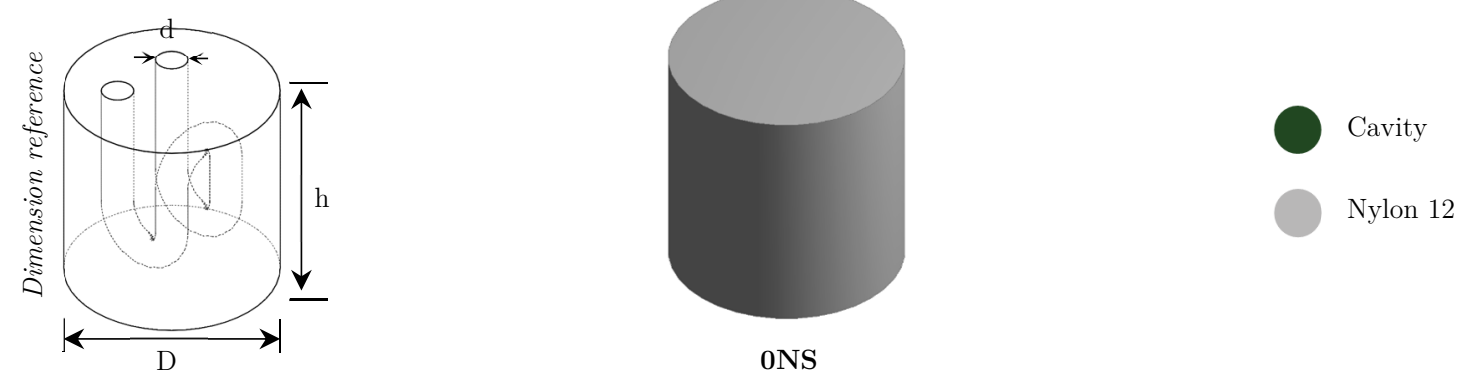

Fig. 4. Design of the test geometries showing both internal and external view. 
To conceive the HQA waveguides featuring three $\Delta l$ and incorporating the four diameters, various orientations of the waveguide were considered. Finally, a simple shape resembling the alphabet $U$ was selected because of its scalable simplicity. This shape was also beneficial when it comes to the powder removal from the waveguides post selective laser melting [44]. However, to quantify the impact of such an orientation, alternative designs designated $\mathrm{H}$ (single helix) and I (double helix) were considered featuring interference length A. Due to the shape constraints of these waveguides, a diameter of $2 \mathrm{~cm}$ was the largest that could be incorporated.

As none of the designs featured a two-tube concept as prescribed by HQ, it was significant to evaluate whether the waveguides are operating as quarter-wave resonators. Consequently, a new design was conceived following the quarter-wave theory to allow for resonance at odd multiples of $100 \mathrm{~Hz}$. The cavity was designated as 1.5DQ and featured a length of $8.58 \mathrm{~cm}$ and diameter of $1.5 \mathrm{~cm}$. This design was held suitable as all the frequencies considered in this study will come under odd multiples of $100 \mathrm{~Hz}$. Finally, a reference solid flat specimen was used to compare the improvement in absorption and TL from the waveguides alone. All design parameters are listed in Table 1 with the resulting test specimen as shown in Fig. 4.

\subsection{Rapid prototyping}

Based on the Computer Aided Design (CAD) geometries, test specimens were rapid prototyped using the SLM process which is one of the leading techniques in Additive Layer Manufacturing (ALM). Among the various ALM techniques, laser melting of polymers has been recognised to be the most promising due to its ability to produce geometrically complex and dimensionally accurate parts with good mechanical strength [45].

Table 2. Nylon-12 material properties used for the laser melting process.

\begin{tabular}{ll}
\hline Properties & Values \\
\hline Density of Laser Sintered Part & 0.9 to $0.95 \mathrm{~g} / \mathrm{cm}^{3}$ \\
Tensile Modulus & $1700 \pm 150 \mathrm{MPa}$ \\
Tensile Strength & $45 \pm 3 \mathrm{MPa}$ \\
Elongation at Break & $20 \pm 5 \%$ \\
Flexural Modulus & $1240 \pm 130 \mathrm{MPa}$ \\
Melting Point & 172 to $180^{\circ} \mathrm{C}$ \\
Coefficient of Thermal Expansion & $1.09 \times 10^{-4} / \mathrm{K}$ \\
\hline
\end{tabular}

Based on the digital designs machine parameters were generated for the laser melting process using Nylon 12 with material properties shown in Table 2. Using the machine software, the designs were oriented and arranged on the SLM print platform so as to enable self-supporting and avoid support structures. This resulted in geometrically accurate waveguides avoiding any remnants that could have been introduced by the support-structures. 
On receiving the sliced digital file, the machine warms up with Nylon-12 powder heated to just below melting point. The powder is then dispersed in a thin layer on top of the platform inside of the build chamber. The printer preheats the powder to a temperature just below the melting point making it easier for the laser to increase the temperature of selected regions of the powder bed. The laser then scans a cross sectional slice of the model, fusing the Nylon-12 particles together to create one solid layer. The unfused powder supports the part during printing and eliminates the need for dedicated support structures. The platform lowers by one layer into the build chamber and a re-coater applies fresh powder on top. The laser then continues the process until the structure is fully manufactured. The finished parts are left to cool down gradually inside the printer itself. The cooled parts are then removed from the build chamber and transferred to the cleaning area to clean the excess powder. Fig. 5. Shows the resulting prototypes after cleaning ready for experimental testing.

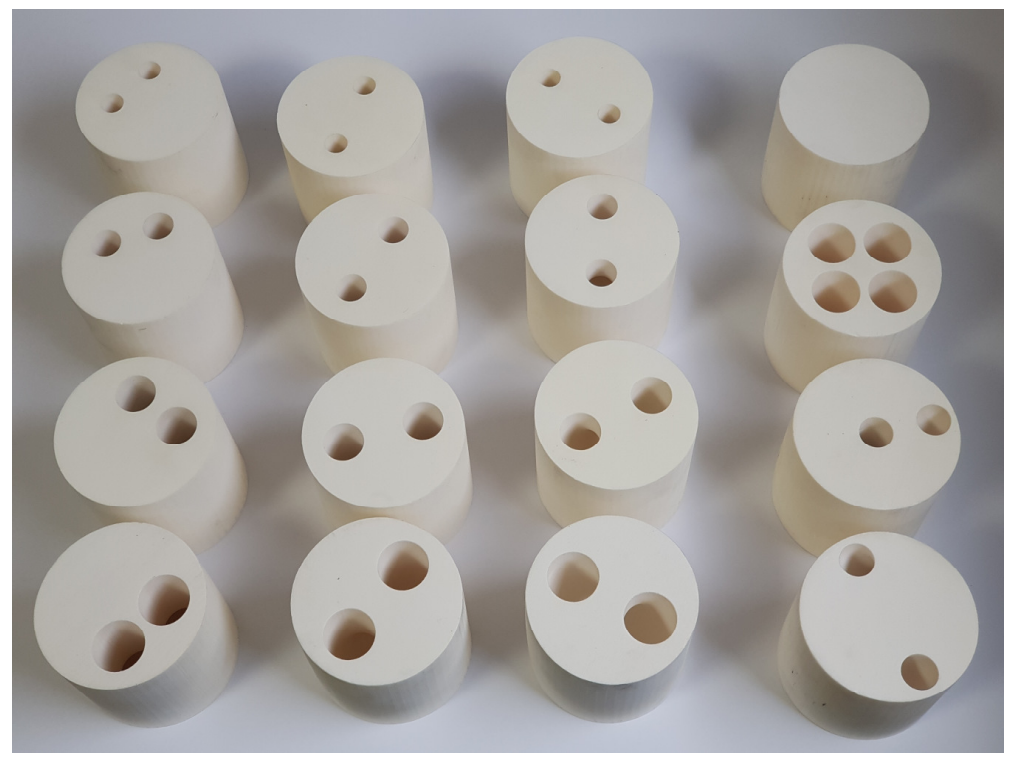

Fig. 5. Nylon-12 experimental test samples manufactured using laser melting.

\subsection{Acoustic testing}

The acoustic testing to measure the sound absorption coefficient $(\alpha)$ and normal incidence transmission loss (TL) were carried out using the SW series Impedance Tubes manufactured by BSWA Tech. The experimental setup uses the transfer function method following relevant standards [46]; where $\alpha$ is measured using the 2-microphone setup and TL uses a four-microphone setup shown in Fig. 6. The procedure for absorption measurement can be found in ISO10534-2 and other literature [47].

A requirement when using impedance tube to characterise the acoustic performance is consideration of lowest cut-off frequency to produce plane waves. In such a way that the resulting sound field in the tube consists of a single component traveling towards and reflected. For the 
test setup considered two microphones (M1 and M2) were mounted in the source chamber (SC) and other two microphones (M3 and M4) were mounted in the receiving chamber (RC). The arrangement meant that both incident and reflected acoustic waves could be obtained. All microphones used were class 1 featuring a frequency response range of 0.02 to $12.5 \mathrm{kHz}$ approximately. A loudspeaker connected to an amplifier was used as the sound source situated at the far end of SC.

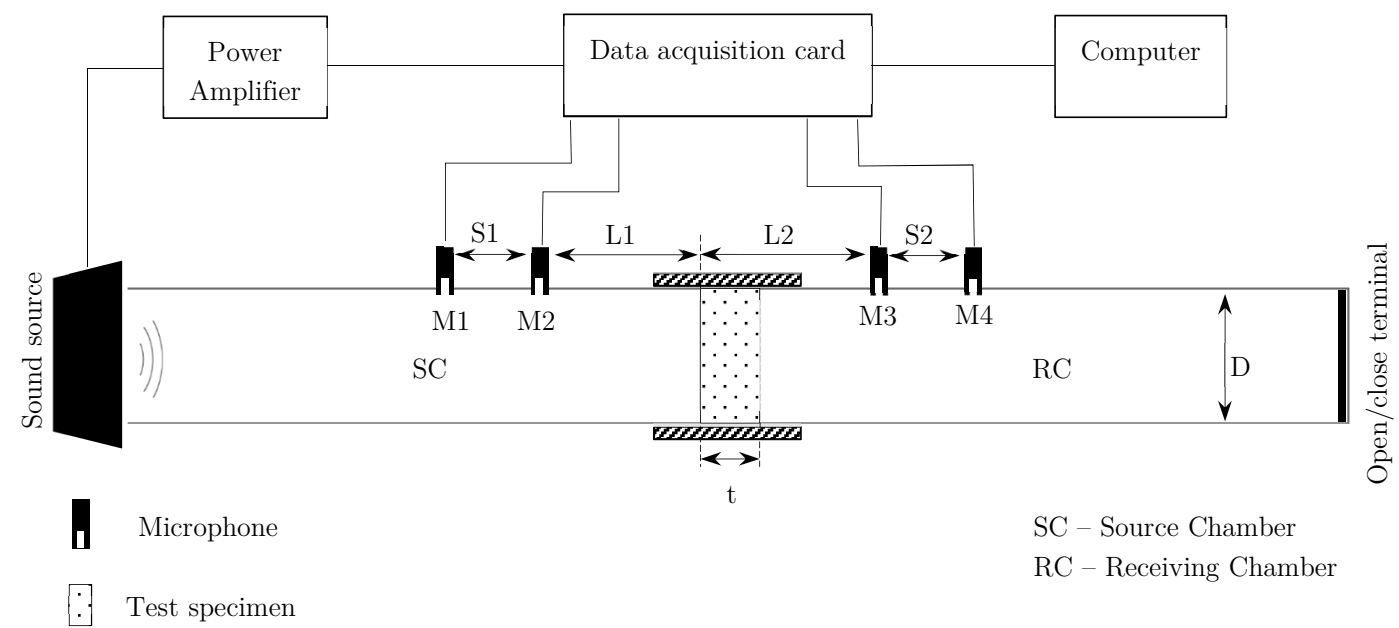

Fig. 6. Experimental setup used to measure the normal incidence transmission loss.

The experimental rig for the TL was coupled to a tone generator through an amplifier and the microphones were connected to a data logger. Using the front surface of the test specimen facing the sound source as reference the relative position of microphone L1, L2, S1 and S2 were measured. Consequently, the complex acoustic pressures at the four microphone locations within the impedance tube can be expressed using Eqn. (3-6).

$$
\begin{aligned}
& P_{1}=\left(A e^{-j k x_{1}}+B e^{j k x_{1}}\right) e^{j \omega t} \\
& P_{2}=\left(A e^{j k x_{2}}+B e^{-j k x_{2}}\right) e^{j \omega t} \\
& P_{3}=\left(A e^{-j k x_{3}}+B e^{j k x_{3}}\right) e^{j \omega t} \\
& P_{4}=\left(A e^{j k x_{4}}+B e^{-j k x_{4}}\right) e^{j \omega t}
\end{aligned}
$$

Where $x_{1}, x_{2}, x_{3}$, and $x_{4}$ are distance from the specimen reference to microphones $M 1, M 2, M 3$ and $M 4$ respectively. Furthermore, $k$ represents the wave number, $A$ and $B$ indicate the incident and the reflected acoustic components in SC and $\mathrm{C}$ and $\mathrm{D}$ indicate the transmitted and the reflected acoustic components in RC. Eqn. (3-6) can then be rearranged to obtain Eqn. (7-10) which can be used to solve for parameters A to D:

$$
A=\frac{j\left(P_{1} e^{j k x_{1}}-P_{2} e^{j k x_{1}}\right.}{2 \sin k\left(x_{1}-x_{2}\right)}
$$




$$
\begin{gathered}
B=\frac{j\left(P_{2} e^{-j k x_{1}}-P_{1} e^{-j k x_{2}}\right.}{2 \sin k\left(x_{1}-x_{2}\right)} \\
C=\frac{j\left(P_{3} e^{j k x_{4}}-P_{4} e^{j k x_{3}}\right.}{2 \sin k\left(x_{3}-x_{4}\right)} \\
D=\frac{j\left(P_{4} e^{-j k x_{3}}-P_{3} e^{-j k x_{4}}\right.}{2 \sin k\left(x_{3}-x_{4}\right)}
\end{gathered}
$$

In order to obtain a reliable solution, the measurements were carried out under two difference boundary conditions. This involves having two end conditions (represented using subscripts 1 and 2 for $\mathrm{A}, \mathrm{B}, \mathrm{C}$ and $\mathrm{D}$ ), for example having a rigid termination (RC closed) or no termination (RC open), then Eqn. (11) can be solved to obtain $\alpha$ :

$$
\alpha=\frac{A_{1} D_{2}-A_{2} D_{1}}{C_{1} D_{2}-C_{2} D_{1}}
$$

Where, $A_{1}, C_{1}, D_{1}$ and $A_{2}, C_{2}, D_{2}$ are the acoustic wave amplitudes for when measurements were taken with the closed and open boundary respectively. Consequently, the transmission loss matrix relates the forward and backward travelling acoustic wave using Eqn. (12).

$$
\left\{\begin{array}{l}
A \\
B
\end{array}\right\}=\left[\begin{array}{ll}
\alpha & \beta \\
\gamma & \delta
\end{array}\right]
$$

Where $\alpha$ and $\delta$ are the pressure transmission loss coefficient of the waveguide (measurement sample) determined from the source and receiving chamber respectively. $\beta$ and $\gamma$ are parameters determining the acoustic impedance of the waveguide. The thus determined transmission loss coefficient $\alpha$ is then used to calculate the TL in decibels using Eqn. (13).

$$
T L=-20 \log (\|\alpha\|)
$$

Before testing the TL values of the waveguides, the microphones were calibrated, and validation test performed using a polyurethane (PUR) specimen of known $\alpha$ and TL values. The relative humidity and temperature of the acoustic lab was maintained at a constant $21^{\circ} \mathrm{C}$ and $64 \%$ respectively through the test for consistency. For each HQA waveguide, six measurements were taken, three at open and three at closed boundary conditions. The data was collected at onetwelve octave band at a frequency range of 400 to $1600 \mathrm{~Hz}$.

\section{Results and discussion}

\subsection{Validation}


Before characterising the acoustic performance of the HQA waveguides, the validation of the experimental right was carried using a material of known $\alpha$ and TL. This was done to corroborate the compliance of the measurement data with ISO10534-2 [46] and to quantify the extent of uncertainty, repeatability and reproducibility of the experimental setup. A $25 \mathrm{~mm}$ thick PUR foam $\left(\rho=6 \mathrm{~kg} / \mathrm{m}^{3}\right)$ was chosen as the validation specimen.

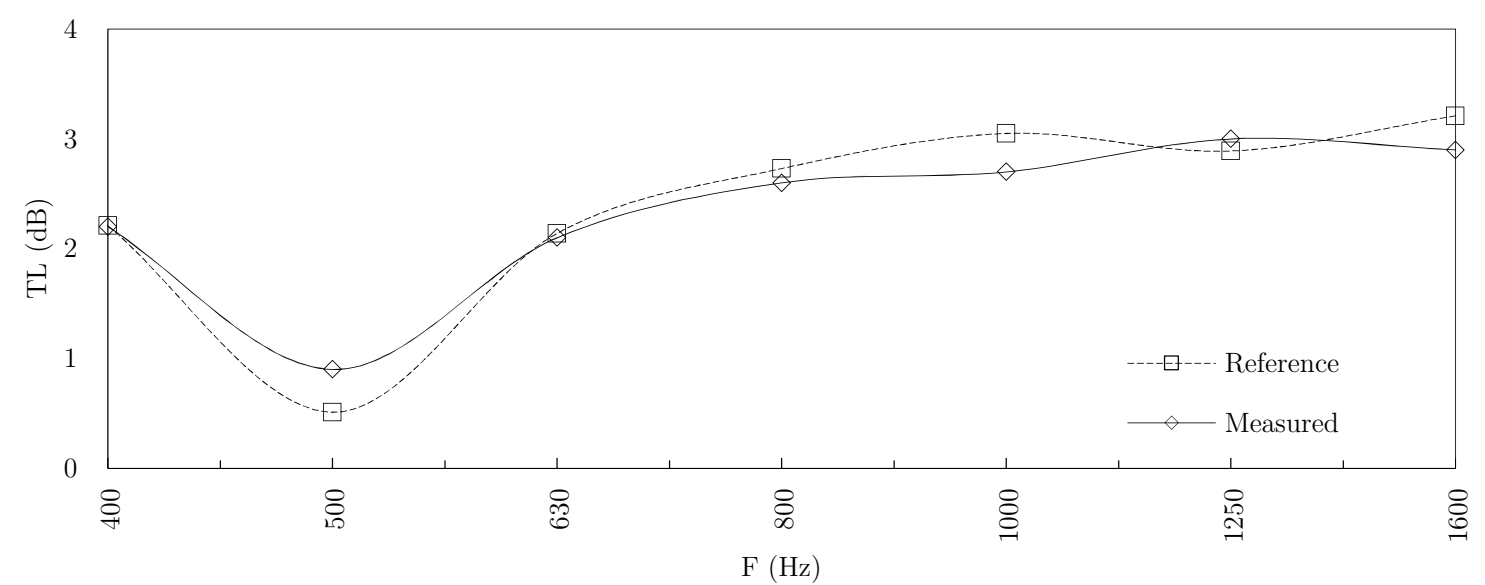

(a)

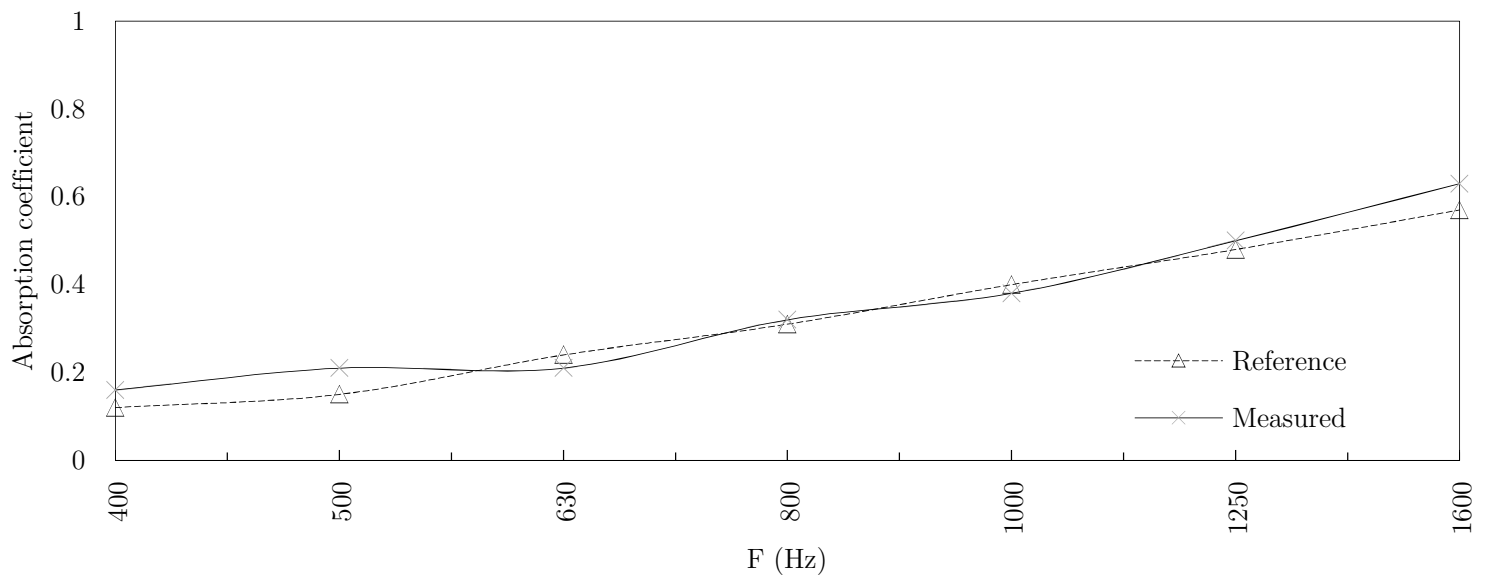

(b)

Fig. 7. Reference and measured values for (a) transmission loss and (b) absorption coefficient.

The two central parameters describing the acoustic insulation of a component are the normal incident sound absorption coefficient $(\alpha)$ and transmission loss (TL). Accordingly, the validation specimen was used to establish the accuracy of both parameters. A frequency range of 400 to $1600 \mathrm{~Hz}$ was deemed appropriate as this accommodated the whole range of $\Delta l$ considered in this study. Fig. 7 presents comparison between acoustical test results and the reference measurements from independent tests at $1 / 3$ octave band. The highest difference observed was $0.39 \mathrm{~dB}$ at 500 $\mathrm{Hz}$ for TL as shown in Fig. 7a. This is well below the ISO10140 [48] defined measurement uncertainty as shown in Table 3 .

Table 3 Allowable measurement uncertainty against experimentally measured. 


\begin{tabular}{ccc} 
Frequency $(\mathrm{kHz})$ & Standard $( \pm \mathrm{dB})$ & Measured $(\mathrm{dB})$ \\
\hline 0.250 & 1.5 & -0.19 \\
0.315 & 1.5 & -0.02 \\
0.400 & 1.2 & +0.01 \\
0.500 & 1.2 & -0.39 \\
0.630 & 1.2 & +0.04 \\
0.800 & 1.0 & +0.13 \\
1.000 & 1.0 & +0.35 \\
1.250 & 1.0 & -0.11 \\
1.600 & 1.0 & +0.31 \\
\hline
\end{tabular}

Similarly, for absorption coefficient, the highest difference between the data sets was $0.06(6 \%)$. Generally, an uncertainty in the measurement of $10 \%(0.1)$ is allowed [49]; consequently, the comparison presented in Fig. 7b is well within acceptable limits. Relating $\alpha$ to TL, a $10 \%$ difference in absorption translates to an uncertainty of approximately $0.50 \mathrm{~dB}$ which is well below the acceptable uncertainty [49] within the frequency range considered. Consequently, the test setup can be considered validated in accordance with relevant standards.

\subsection{Absorption}

\subsubsection{Noise cancellation at selected frequency}

The primary focus was the validation of the hypothesis to assess HQA waveguide's capacity to create DI and in turn passive noise cancellation. At $\Delta l / l=1$, the waveguide 1.5AU was designed to create DI and thus noise cancellation at 0.5 and $1.5 \mathrm{kHz}$ at $\mathrm{m}=1$ and 2 respectively. Analysing the results at 1/12 octave band as shown in Fig. 8, substantial increase in $\alpha$ ( 0.96 and 0.98 for $m=1$ and 2 respectively) was observed at 0.475 and $1.4 \mathrm{kHz}$ demonstrating almost complete noise cancellation. However, at $m=1$, a $-25 \mathrm{~Hz}$ variation between the theoretical and experimental frequency was observed where the highest $\alpha$ was observed. This may be due to the HQA's single channel waveguide as opposed to a traditional two channel setup. In addition, any change in the length of waveguide due to tortuosity (the twisted nature of the waveguide) was not adjusted for in the computation of the $\Delta l / l$ ratio that may have also been a contributing factor.

A comparison of the performance of the HQA waveguide in relation to a fully solid flat Nylon12 geometry 0NS is also carried out (Fig. 8). This was done to evaluate whether the absorption results obtained from the waveguide has any correlation with the acoustic modes of the experimental setup or the material used. The results clearly show that when a flat specimen was used there were no significant spikes in $\alpha$ values instead the values seems to coincide with the surface absorption of Nylon-12. This shows that the waveguide design itself is the contributing factor towards the noise cancellation and hence the heightened $\alpha$ values. 


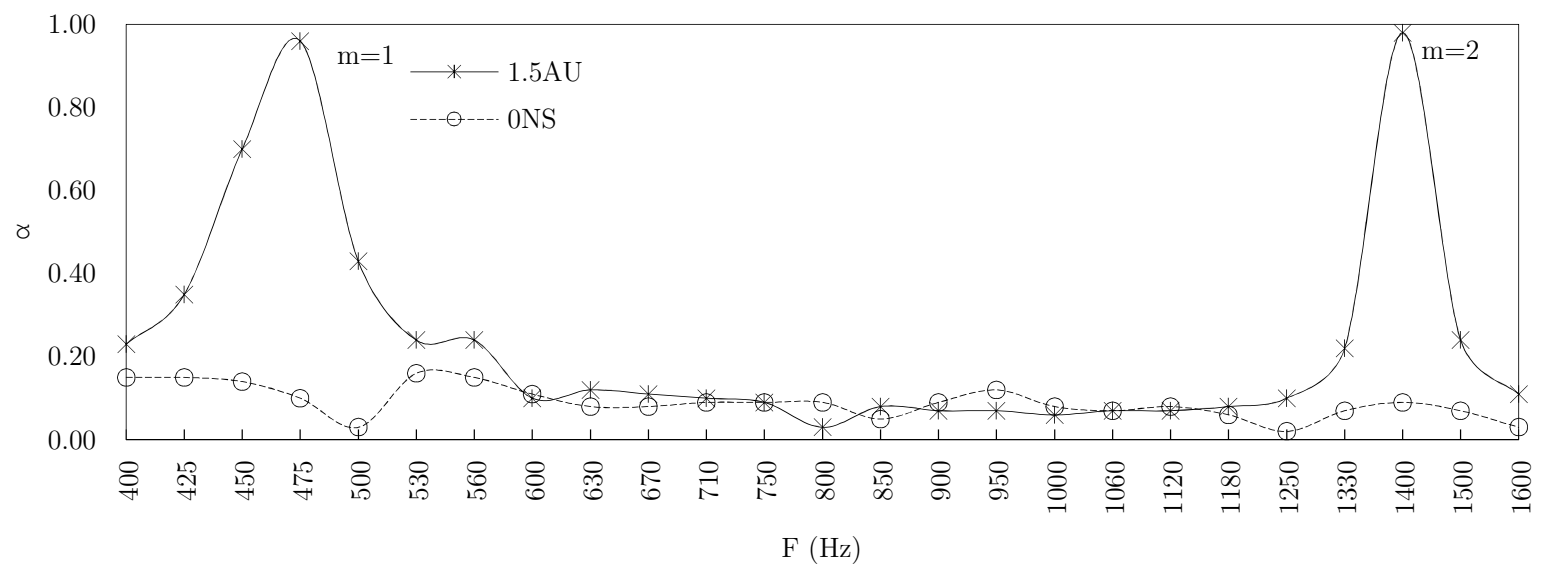

Fig. 8. Sound absorption coefficient for interference cavity 1.5AU in comparison with solid specimen 0NS.

It was also encouraging to see that the flat specimen and the waveguide showed similar $\alpha$ values in the non-targeted zone. This evidences that the HQA waveguide is performing as hypothesised and can achieve frequency dependent noise cancellation with minimal influence on the nontargeted frequencies. Consequently, the $\alpha$ spikes of 0.96 at 0.475 and 0.98 at $1.4 \mathrm{kHz}$ can be related to the occurrence of DI starting at $m=1$ and 2 in a positive integral manner as linked in Eqn. (1). The absorption values close to unit shows that complete absorption of the sound waves is taking place at selected frequency demonstrates the effectiveness of the HQA waveguide in comparison to other resonance-based systems.

\subsubsection{Effect of 'waveguide diameter to transmission-length-difference' ratio}

For a logical and coherent discussion, the data from the experimental tests are grouped under associated cavity length $(\Delta l)$ and an overarching discussion connecting the results are presented. For every HQA waveguide of type U (AU, BU and CU), four diameters considered were $1.5 \mathrm{~cm}$, $2 \mathrm{~cm}, 2.5 \mathrm{~cm}$ and $3 \mathrm{~cm}$ represented using an alphanumeric system as listed in Table 1 . The diameter $3 \mathrm{~cm}$ was the largest possible to contain the longest waveguide within the experimental samples. The smallest was chosen to be half $(1.5 \mathrm{~cm})$ to identify any potential correlation.

From the results presented in Fig. 9, it can be seen that for the HQA waveguide at $343 \mathrm{~mm}$ at all tested diameters exhibits $\alpha$ spikes representative of noise cancellation at targeted frequencies. However, a steady decline in the absorption coefficient consistent at both $\mathrm{m}=1$ and 2 were observed as the diameter increased. The highest absorption coefficient for the longest waveguide was delivered by the smallest diameter of $15 \mathrm{~mm}$. Despite the elaborated selection of diameters, it is not known at this point whether a further reduction in the diameter is capable of delivering high $\alpha$ at the selected frequencies. Therefore, further work in this aspect is necessary to identify the smallest diameter capable of the highest $\alpha$ for the HQA waveguide. Nevertheless, the trend in the result is promising and shows that HQA waveguides can be manufactured at smaller size 
to tackle low frequency acoustic emission which if of significant advantage to the acoustic community.

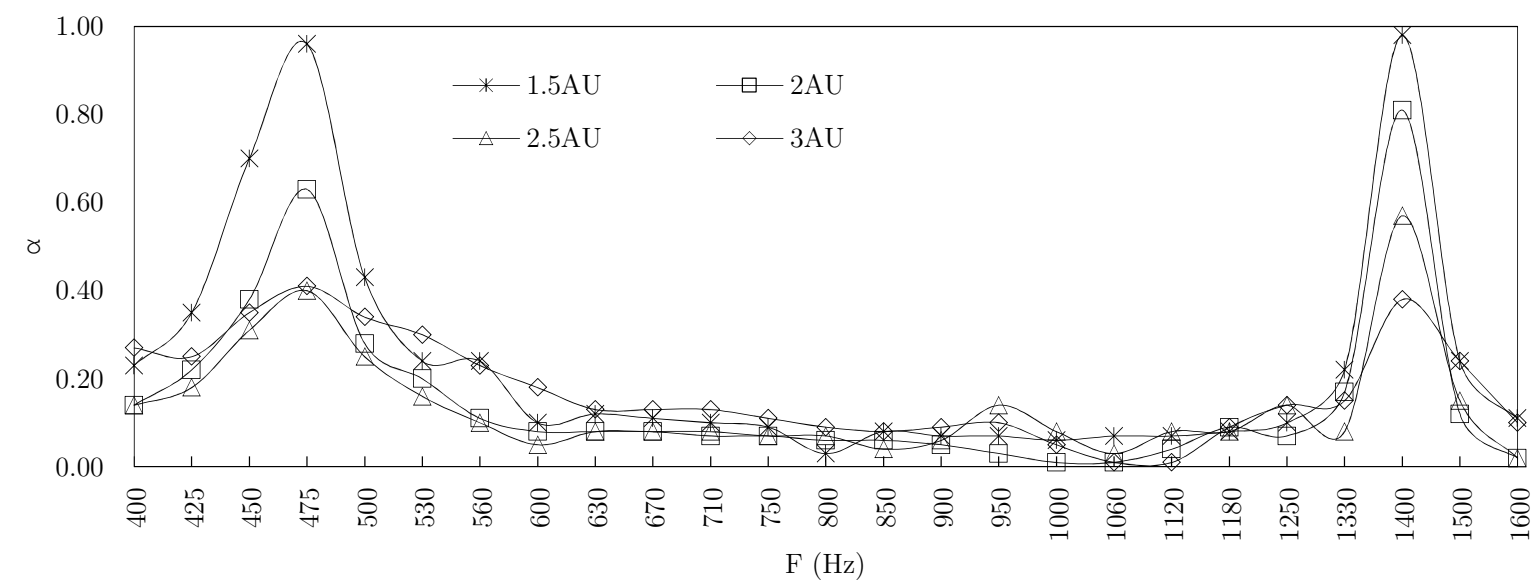

Fig. 9. Effect of diameter on the absorption coefficients of U type HQA waveguides featuring a $\boldsymbol{\Delta} \boldsymbol{l}$ of $34.3 \mathrm{~cm}$.

Fig. 9 clearly shows a coherent pattern of decreasing $\alpha$ both at $m=1$ and 2 at interference frequencies as a result of the increase diameter. The pattern is particularly distinctive at $m=2$ where the $3 \mathrm{~cm}$ dia. shows the smallest $\alpha$ and every $5 \mathrm{~mm}$ decrement in diameter resulted in consistent improvements reaching an $\alpha$ of 0.98 at the smallest diameter. Poor $\alpha$ at larger dia. can be due to the influence of multiple acoustic reflection facilitated by the relatively large crosssection of the waveguide. While the influence of the cross-sectional area on the acoustic performance of HQA need further evaluation, $\alpha$ is still higher compared to the surface absorption of Nylon-12.

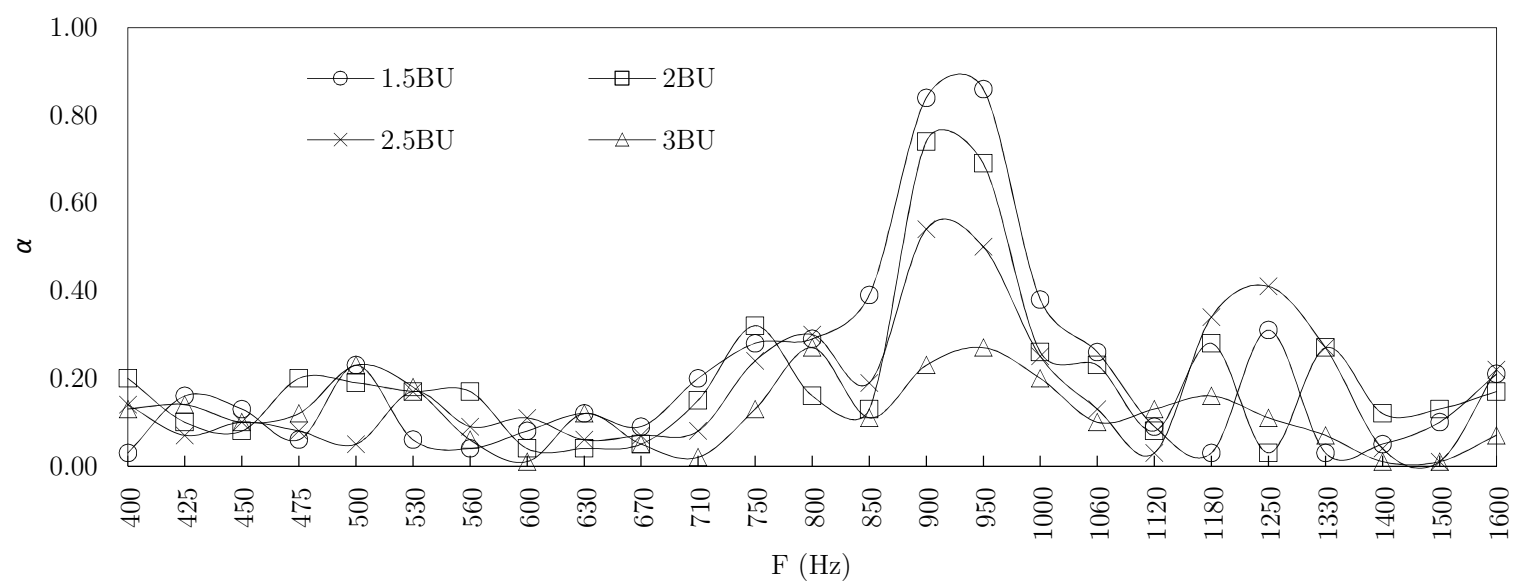

Fig. 10. Effect of diameter on the absorption coefficients of type $\mathrm{U}$ waveguides with a $\Delta l$ of $17.15 \mathrm{~cm}(\mathrm{~B})$.

Consistent with AU, the HQA waveguides characterised under the category BU were also successful in delivering noise cancellation at the desired frequency as shown in Fig. 10. The $\alpha$ spikes as a result of the waveguide length occurred at a frequency of $0.950 \mathrm{kHz}$ for $m=1$. This accounts for a difference of $50 \mathrm{~Hz}$ between the predicted $(1 \mathrm{kHz})$ and experimental frequency accounting for a $5 \%$ error. However, the shift $5 \%$ shift in frequency is consistent with previous 
results obtained for AU. For example, when the sound transmission-length-difference was halved $(34.3 / 2=17.15 \mathrm{~cm})$, the frequency doubled $(475 \times 2=950 \mathrm{~Hz})$. Accordingly, the reason for this is the small changes in length due to the added tortuosity of the waveguides which was not accounted in the theoretical prediction.

Additionally, $\alpha$ spikes due to DI was observed to vary significantly depending upon the diameter of the waveguide as shown in Fig. 10. The highest $\alpha$ BU was exhibited by the waveguide that featured the smallest $d$, a trend similar to AU. Furthermore, $\alpha$ was found to decrease with every $5 \mathrm{~mm}$ increment in $d$. In addition to the $\alpha$ spike at the design frequency inconsistent absorption spikes averaging 0.32 where observed around $1.250 \mathrm{kHz}$. It is unclear from the data whether the additional spikes are a function of the waveguide alone or due to a coupling loss as a result of the impedance chamber. While the additional $\alpha$ spikes are beneficial to the overall sound absorption performance of the waveguide, further studies are necessary to refine the 1D theory to account of unpredicted effects.

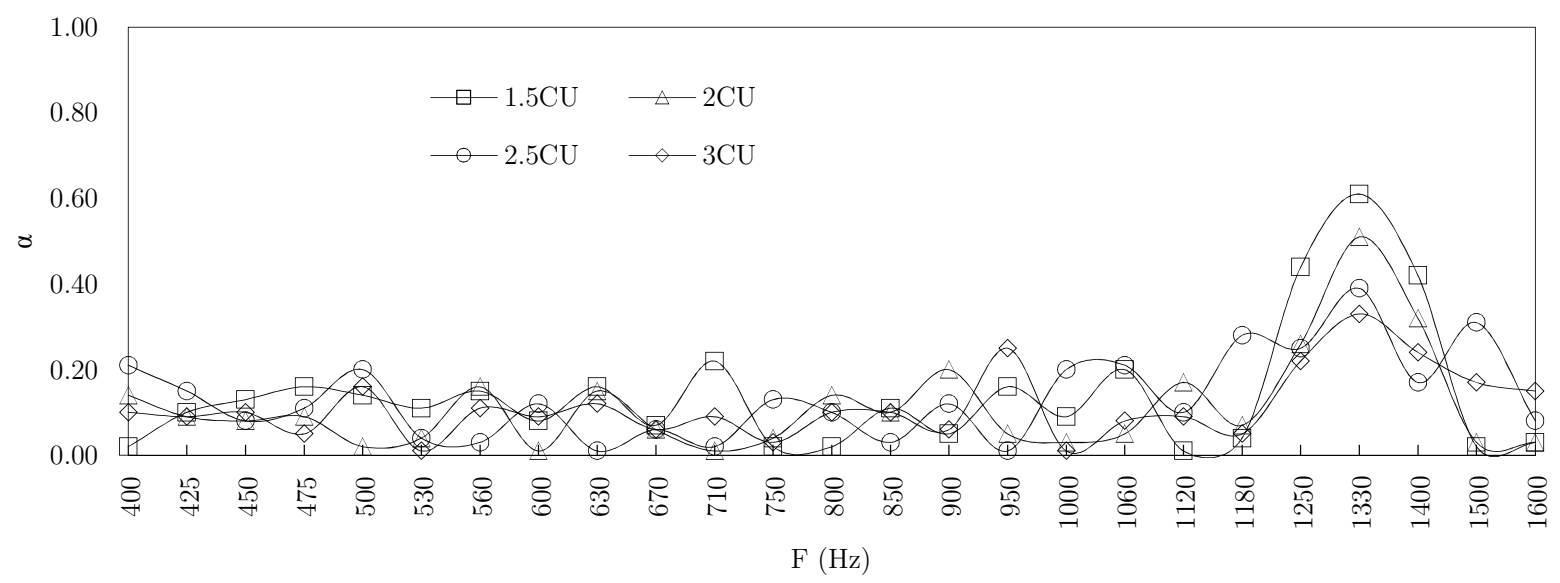

Fig. 11. Effect of diameter on the absorption coefficients of type $\mathrm{U}$ waveguides with a $\Delta l$ of $11.43 \mathrm{~cm}(\mathrm{C})$.

Fig. 11. shows the acoustic performance of the HQA waveguide characterised under UC featuring the smallest $\Delta l$. The $\alpha$ spikes due to noise cancellation occurred at $1.330 \mathrm{kHz}$ in comparison to the design frequency of $1.5 \mathrm{kHz}$, a difference of $12 \%$. This shows that for higher frequencies the difference between the theoretical and experimental frequencies exhibits a wider shift. This was expected as small changes in $\Delta l$ has significant influence as the wavelength decreases. Looking at the influence of the waveguide dia., the trend was similar to the previous waveguides where the best performance was exhibited by the smallest $d$. However, as the acoustic wavelength decreased, waveguides that feature comparable cross-section started experiencing multidimensional interference and resonance phenomenon delivering numerous low-level $\alpha$ spikes. This is clear evidence that the geometries of the HQA waveguides need optimisation both in size shape and tortuosity to create the most controlled acoustic environment. 
The highest acoustic absorption by the best performing HQA waveguides as a result of controlled wave interference under all the different $\Delta l$ is shown in Fig. 12. For all HQA design, a diameter of $15 \mathrm{~mm}$ (smallest) showed the best performance. The longest waveguide showed almost complete noise cancellation resulting in $\alpha \approx 1$ at both $m=1$ and 2 . However, as the $\Delta l$ values decreased so did the noise cancellation with $\alpha$ reaching 0.61 at $11.43 \mathrm{~cm}$. This shows the importance of 'interference length to diameter' ratio in the design of HQA waveguides where the influence of diameter increases significantly as the frequency increases. Nevertheless, the reduction in peak $\alpha$ values were consistent and corelated with the step-by-step decrease in $\Delta l$.

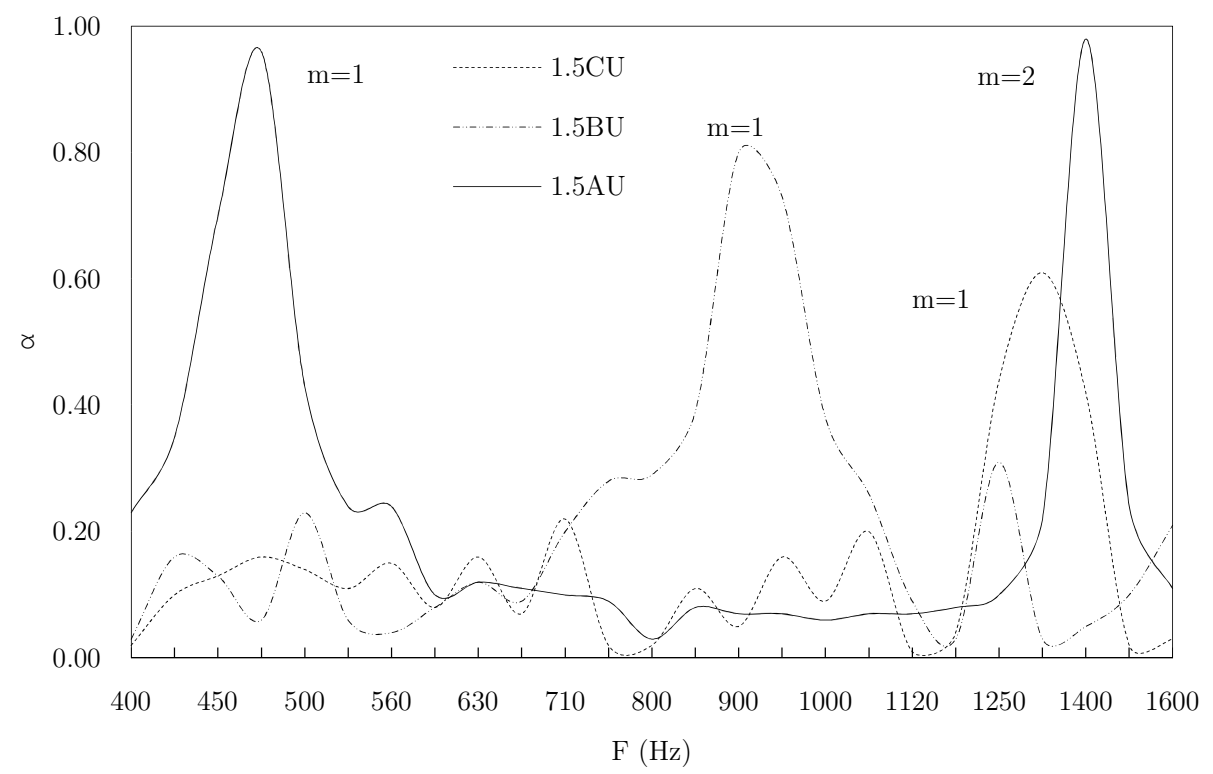

Fig. 12. Absorption due to acoustic wave interference exhibited by best performing HQA waveguides.

When the sound transmission-length-difference of the HQA waveguide was halved from A to B and then to C, effective noise cancellation fell by $\sim 20 \%$ each time. Spatial interference as a result of the reducing acoustic wavelengths enabled by the comparatively large cross-sectional area of the waveguide can be the reason for this. Accordingly, HQA waveguides featuring smaller $d$ is demanded for complete cancellation at higher frequencies. This is a critical finding and add to the one-dimensional modified theory of HQ tubes utilised for the design of these geometries. Furthermore, the width of the $\alpha$ peaks as a result of wave interference at $m=1$ are similar for all the HQA waveguide designs considered. This is crucial in designing multifrequency waveguides within a single geometry.

\subsubsection{Effect of waveguide tortuosity}

Tortuosity here relates to the twisted nature of waveguide within the cylindrical volume. It was significant to identify whether the noise cancellation had a relationship linking to the tortuosity of the HQA waveguide. In order to study this phenomenon in isolation, two different tortuous HQA waveguides $\mathrm{AH}$ and $\mathrm{AI}$ were developed. The waveguides AU, AH and AI share identical 
$\Delta l$ and $d$ parametric values of 34.3 and $2 \mathrm{~cm}$ respectively. However, the tortuous nature of the waveguides are dissimilar as can be seen from Fig. 4 . While the waveguide AU represents a relatively simple geometry featuring one bend, $\mathrm{AH}$ and $\mathrm{AI}$ feature significant tortuosity resembling a spiral and twin-spiral waveguide respectively.

Comparing the results as shown in Fig. 13, despite the change in waveguide tortuosity, noise cancellation was observed at the predicted frequencies both at $m=1$ and 2 . Nevertheless, the associated $\alpha$ spikes showed a slight difference between the waveguides. The twin spiral waveguide AI showed an absorption of 0.73 at $m=1$ as opposed to 0.63 for both $\mathrm{AU}$ and AH. However, at the $2^{\text {nd }}$ integral spike both $\mathrm{AU}$ and $\mathrm{AI}$ exhibited similar performance showing an $\alpha$ of 0.84 , a difference of 0.12 .

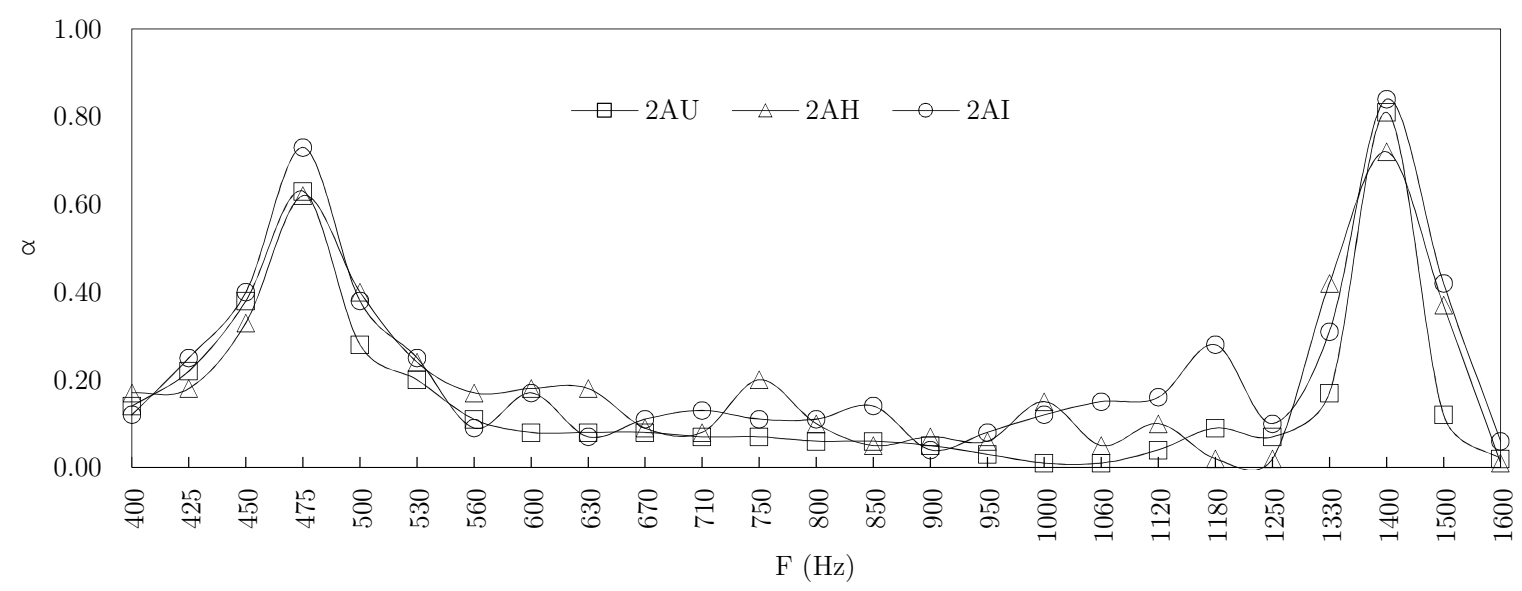

Fig. 13. Effect of waveguide tortuosity on the measured absorption coefficients.

Overall when it comes to the tortuous nature of the HQA waveguides no difference in the predicted frequency of noise cancellation were observed in this study. Furthermore, difference in $\alpha$ due to tortuosity was found to be minimal and shows to demonstrate that the HQA concept allows for a design freedom allowing the full utilisation of confined spaces. However, it is acknowledged that the tortuosity of HQA waveguide requires further studies to effectively characterise its influence on $\alpha$.

\subsubsection{Comparison to Quarter-wave resonators}

The one-dimensional interference theory adopted for HQA waveguides were linked to the length; a phenomenon that can be considered similar to quarter-wave resonators. Therefore, it was significant to identify whether the performance of the HQA waveguide could be linked to the design guidelines associated with traditional quarter-wave resonators. Accordingly, a new design 1.5DQ was developed featuring quarter-wave resonators instead of interference waveguides. A diameter of $1.5 \mathrm{~cm}$ was chosen, as it was the best performing diameter in all the HQA waveguides tested. A total of four quarter-wave waveguides were built into a single sample, each featuring a length of $8.58 \mathrm{~cm}$ to accommodate the largest length of $34.3 \mathrm{~cm} \mathrm{(A).} \mathrm{At} \mathrm{a} \mathrm{length} \mathrm{of} 8.58 \mathrm{~cm}$, 
the quarter-wave tubes were expected to exhibit resonance at odd multiples of $100 \mathrm{~Hz}$ (i.e. 500 $\mathrm{Hz}$ at $\mathrm{n}_{\mathrm{R}}=3,700 \mathrm{~Hz}$ at $\mathrm{n}_{\mathrm{R}}=4,900 \mathrm{~Hz}$ at $\mathrm{n}_{\mathrm{R}}=5$ etc.).

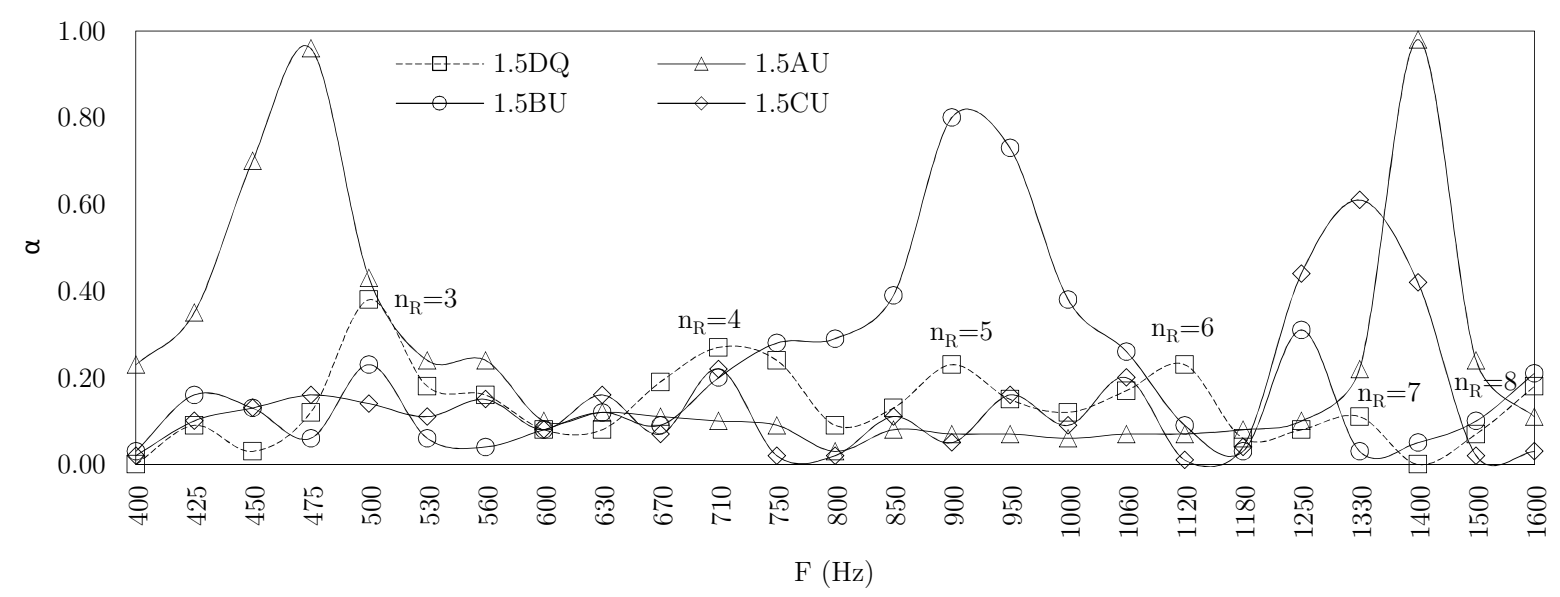

Fig. 14. Comparison between HQA waveguide and quarter-wave resonators of similar radial dimensions.

Comparison of the performance shown in Fig. 14 shows that the absorption for HQA waveguide was substantially higher than quarter-wave resonators. Even though, resonance peaks where consistently observed at every odd multiples of $100 \mathrm{~Hz}$, the highest absorption was limited to 0.38 at $\mathrm{n}_{\mathrm{R}}=3$. Therefore, the absorption exhibited by the HQA waveguide are due to the phenomenon of sound-interference and cannot be compared to the performance of quarter-wave resonators. It is acknowledged that if a state-of-the-art quarter-wave resonator with optimum geometrical parameters are used, comparable $\alpha$ values to that of HQA waveguides can be reached. However, the reason for the presented comparison is to merely show that the HQA waveguide performs differently to a quarter-wave resonator.

\subsection{Transmission Loss (TL)}

\subsubsection{Targeted creation of $T L$}

There is no prior literature to reference whether geometries delivering interference such as the HQA waveguide can recreate TL peaks corresponding to design frequencies. Consequently, one of the key objectives of this study was to see whether TL peaks related to design frequencies for the HQA waveguides.

Fig. 15 shows that the waveguides under respective $\Delta l$ exhibited DI peaks resulting in superior TL at targeted design frequencies. This was encouraging as design guidelines dictating absorption and sound transmission for partitioned structures are often different. Comparing the performance of HQA waveguides with a flat specimen, significant improvement in TL was observed at all interference waveguides. For the longest transmission-length-difference of $34.3 \mathrm{~cm}(\mathrm{AU})$, the first and second TL interference peaks were observed at 450 and $1400 \mathrm{~Hz}$ corresponding to $53.10 \mathrm{~dB}$ and $64.58 \mathrm{~dB}$ respectively. In comparison to the flat specimen, this was an improvement in TL of $30.15 \%$ and $65.59 \%$ at targeted frequencies of $450 \mathrm{~Hz}$ and $1400 \mathrm{~Hz}$ respectively. 


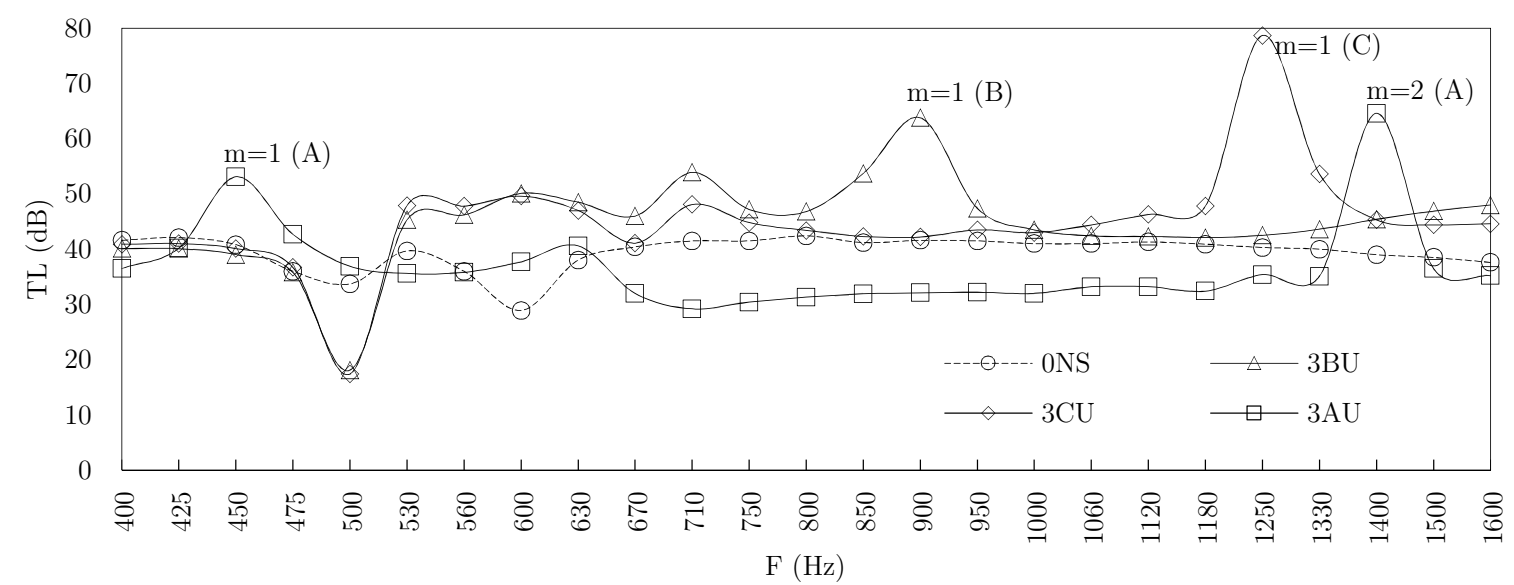

Fig. 15. Transmission loss of best performing HQA waveguides under each $\boldsymbol{\Delta} \boldsymbol{l}$ considered in comparison with a solid flat specimen.

For cavity lengths of $17.15 \mathrm{~cm}(\mathrm{BU})$ and $11.43 \mathrm{~cm}(\mathrm{CU})$ TL peaks due to interference occurred at $900 \mathrm{~Hz}$ and $1250 \mathrm{~Hz}$ resulting in $53.36 \%$ and $95.28 \%$ improvement in comparison to a solid flat specimen. A slight shift in TL interference frequency $(1250 \mathrm{~Hz})$ was observed for design $\mathrm{C}$ compared to the frequency at which absorption peak occurred $(1330 \mathrm{~Hz})$. This shift in $80 \mathrm{~Hz}$ can be attributed to the large diameter of the cavity shifting the overall mass of the component. Other than for the targeted design frequencies, a reduction in TL from $670 \mathrm{~Hz}$ to $1330 \mathrm{~Hz}$ was observed for $3 \mathrm{AU}$ in comparison to solid specimen. This was expected due to a $30.87 \%$ reduction in material mass featured by the cavity $3 \mathrm{AU}$ in comparison to the solid specimen. As the cavity size reduced the overall performance at non-interference frequencies where better than the solid specimen except for $500 \mathrm{~Hz}$ which can be associated as the eigenmode frequency of the specimen $3 \mathrm{BU}$ and $3 \mathrm{C}$.

\subsubsection{Effect of waveguide diameter on TL}

Comparing the results as shown in Fig. 16, all HQA waveguides expect 1.5AU exhibited TL peaks at the first interference frequency of $450 \mathrm{~Hz}(m=1)$. A similar performance was observed at the second interference frequency with 2.5AU showing a slight shift from $1400 \mathrm{~Hz}$ to $1500 \mathrm{~Hz}$. Overall, with the decrease in diameter the TL peaks decreased steadily, a trend contrary to what was observed for absorption. 


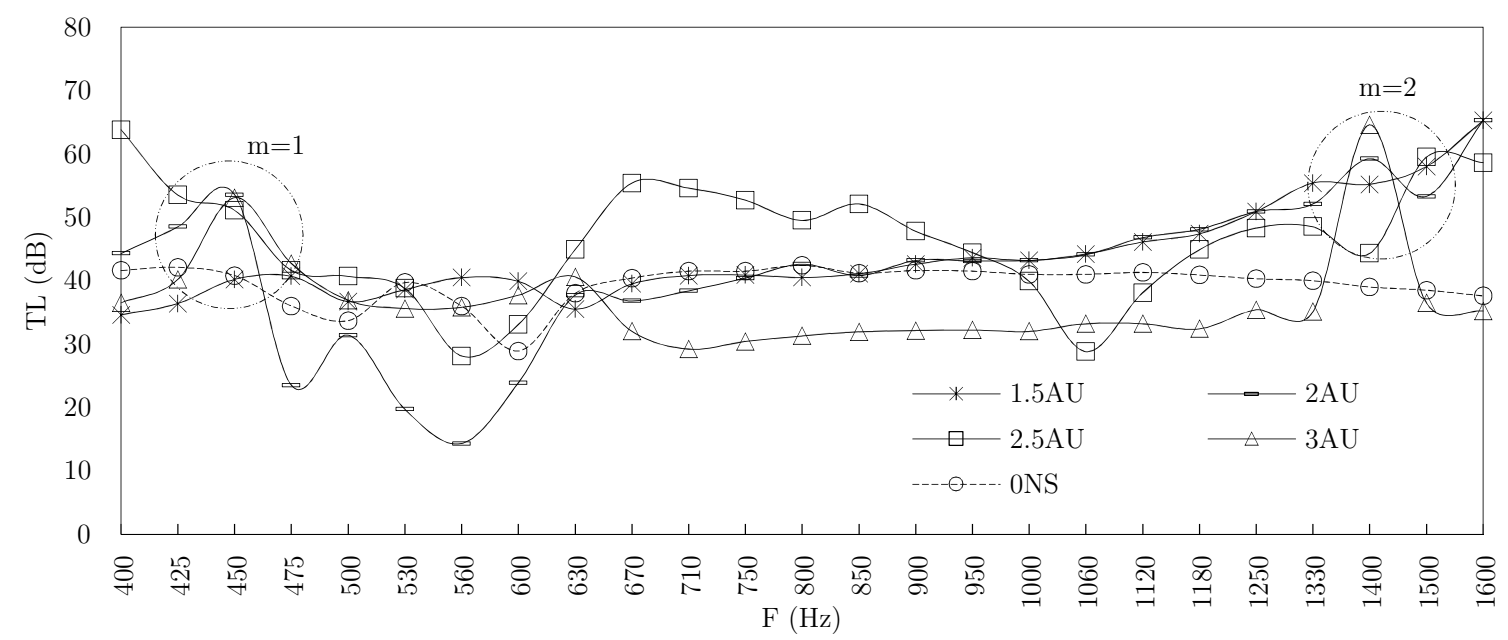

Fig. 16. Effect of diameter on the transmission loss of HQA waveguides with a $\Delta l$ of $34.3 \mathrm{~cm}(\mathrm{~A})$.

As opposed to $\alpha$, the highest transmission loss was found for the waveguide that feature the highest $d$ (3AU) at both $m=1$ and 2. The substantial reduction in the TL amplitude at smaller dia. could be attributed to the 'cavity to non-cavity' material ratio. Nevertheless, the transmission loss behaviour through HQA waveguides needs further investigation to improve the 1D design guideline to take into consideration the diameter for optimal TL performance.

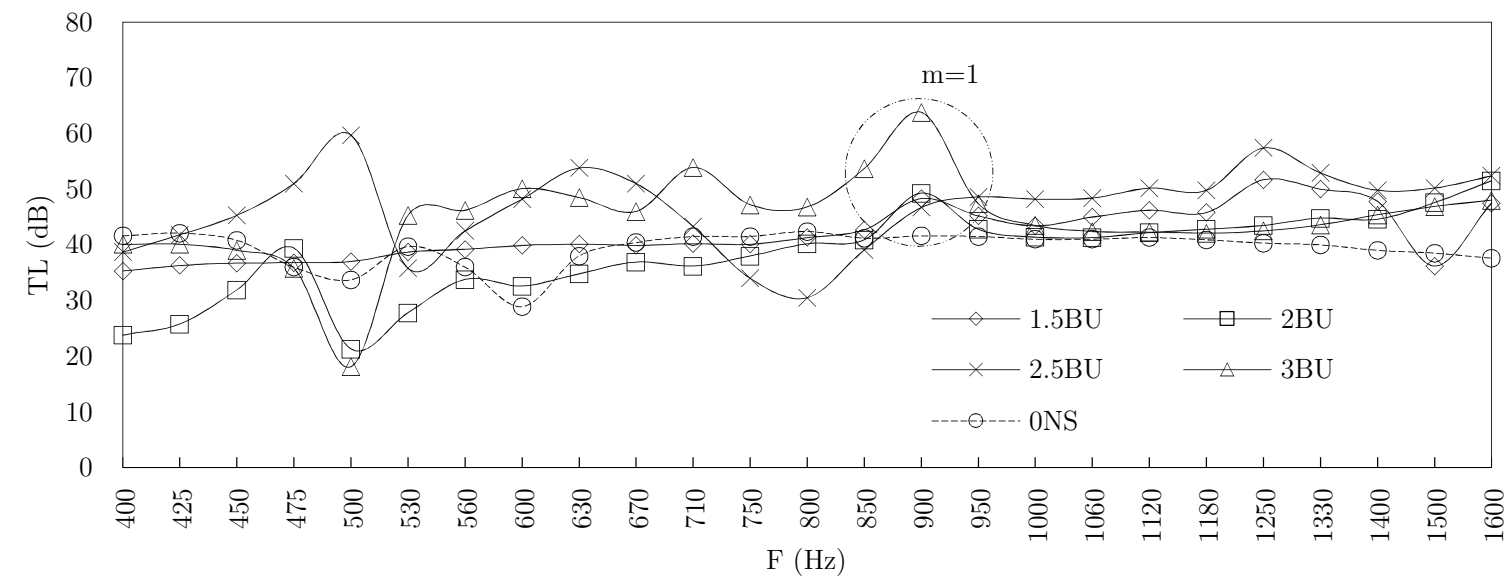

Fig. 17. Effect of diameter on the transmission loss of HQA waveguides with a $\Delta l$ of $17.15 \mathrm{~cm}(\mathrm{~A})$.

Comparing the performance of the waveguides with a solid flat specimen, significant improvement in TL was observed for all HQA waveguides tested. However, for non-interference frequencies, design 3AU and 2.5AU exhibited slightly dissimilar performance between $670 \mathrm{~Hz}$ and $1330 \mathrm{~Hz}$ due to the reduction in material mass contributed by the waveguide volume. As the cavity volume decreased, the performance at these frequencies became comparable to the solid specimen.

Consistent with the performance of the previous designs, all HQA waveguides tested under the category BU also recreated TL peaks as shown in Fig. 17. The TL interference peaks for all the four designs under this category were observed around $900 \mathrm{~Hz}$; a shift of $50 \mathrm{~Hz}$ when compared 
to frequency at which absorption was observed. Comparing the TL curves as shown in Fig. 17, the extend of transmission loss as a result of the waveguide design was highly influenced by $d$. The peak transmission loss as a result of passive noise cancellation from the waveguides were $48.30,49.30,46.70$ and $63.80 \mathrm{~dB}$ at a $d$ of $15,20,25$ and $30 \mathrm{~mm}$ respectively. Consequently, the highest TL at interference coincided with the highest $d$ tested showing an improvement in acoustic insulation of $53.36 \%$ compared to a solid flat specimen. The lowest performance was an improvement of $16.1 \%$ exhibited by the HQA waveguide that featured the smallest $d$. Evaluating the overall performance, it is evident that the difference in material mass have a certain impact on the performance at the non-interference frequencies. As the cavity diameter decreased, the TL curve at non-interference frequencies became more and more comparable to the solid specimen similar to previous designs.

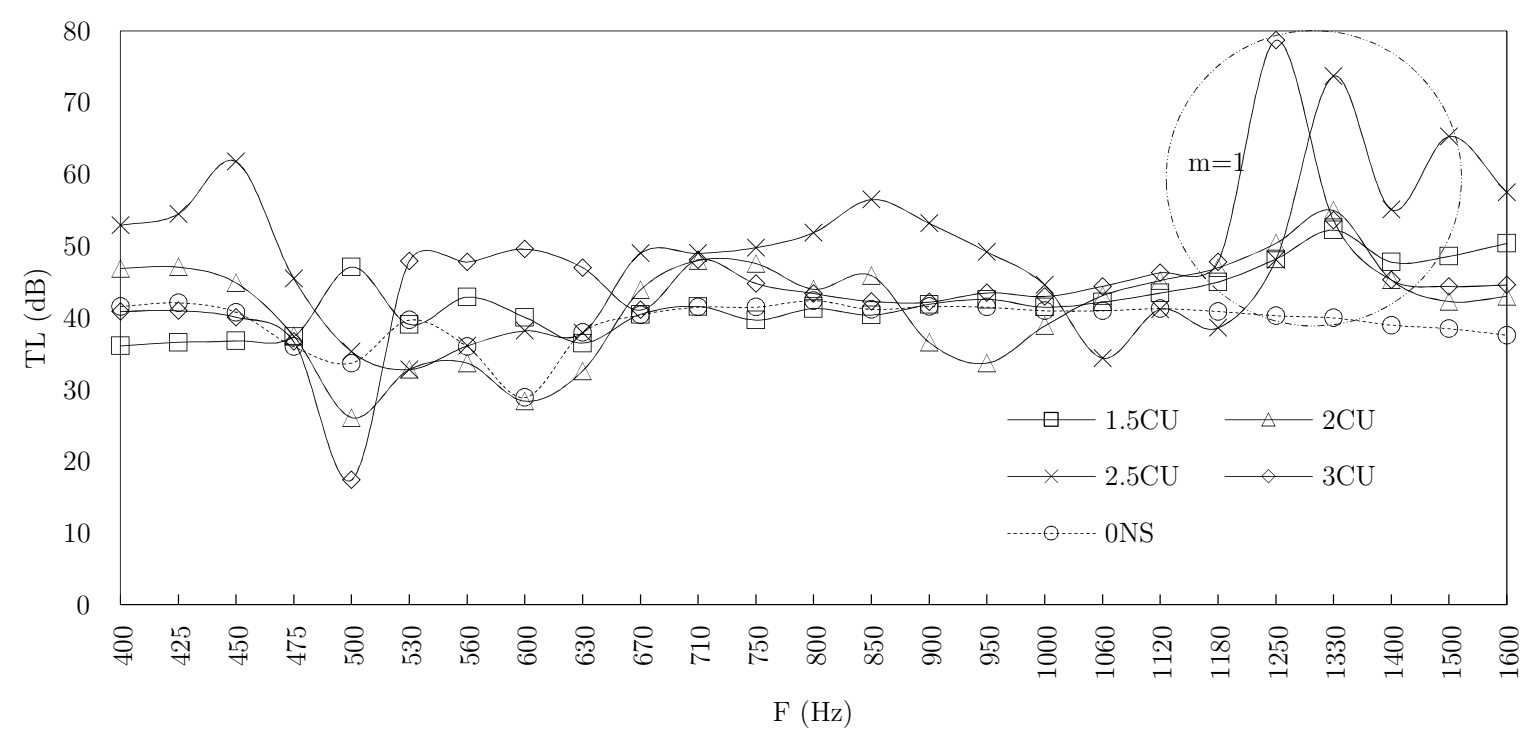

Fig. 18. Effect of diameter on the transmission loss of HQA waveguides with a $\Delta l$ of $11.43 \mathrm{~cm}(\mathrm{C})$.

From the results of the HQA waveguide category CU (Fig. 18), the noise cancellation is comparable to the waveguides in the previous category. However, the frequency of highest noise cancellation changed slightly for the biggest $d$ from 1.330 to $1.250 \mathrm{kHz}$. For the rest of the waveguide diameters grouped under $\mathrm{CU}$, the TL spikes were at $1.330 \mathrm{kHz}$ similar to what was observed for $\alpha$. However, when it comes to the TL performance, the $30 \mathrm{~mm}$ dia. outperformed all other waveguides resulting in a $78.79 \mathrm{~dB}$ acoustic insulation, an improvement of $95.28 \%$ compared to the solid flat sample. The lowest transmission loss due to noise cancellation was exhibited by waveguide that featured a $15 \mathrm{~mm}$ dia. with only an improvement of $30.65 \%$ in comparison to the flat sample.

It is evident that the larger diameter waveguides in comparison are exhibiting numerous TL peaks and valleys throughout the frequency range tested. It is not clear at this point whether all of the spikes can be associated to the phenomenon of acoustic wave interference. Nevertheless, 
the fluctuation can be easily classified due to their low TL amplitude in comparison to the noise cancellation peaks. This is perhaps pointing to the difference that the overall volume of the waveguides might be acting also as resonance devices between when it comes to transmission through them. However, as the diameter of the waveguides become smaller, the performance of the TL curve other than for the predicted frequencies are comparable to the solid specimen.

At all frequencies tested, the largest diameter exhibited the best TL performance; as the design frequency increased so does the amplitude of the TL peaks. This was contrary to absorption curves, as the best performance was observed for the smallest diameter tested. Accordingly, the HQA waveguides should be designed with the potential application of either absorption or transmission. The best performing HQA waveguide for absorption might be the worst performing when it comes to transmission loss. Nevertheless, by the controlling the number of parameters demonstrated in this study required performance can be achieved.

The presented HQA waveguide designs resulted in an improvement in the sound transmission loss of $95.28 \%, 53.36 \%$ and $30.15 \%$ under groupings $\mathrm{C}, \mathrm{B}$ and $\mathrm{A}$ respectively. These are substantial improvements difficult to be achieved through traditional mass or stiffness manipulation showcasing the potential of interference-based TL improvement that can be employed for noise abatement. For the longest $\Delta l$ tested, the performance of the TL curve improved from $30.15 \%$ to $65.59 \%$ from first to the second interference frequency showing that further research is needed to identify the optimal 'm' value for design guidelines. Nevertheless, the methodology for improved TL demonstrated through HQA waveguide is suitable for incorporation into building walls or noise barrier to improve acoustic insulation at the same time reducing material mass.

\subsubsection{Effect of waveguide tortuosity on $T L$}

The designs 2AU, AH and AI all share the same waveguide length and diameter; a combination that was chosen to allow for alternative waveguide tortuosity within the noise cancellation frequency. Comparing the results as shown in Fig. 19, all waveguides show transmission loss as a result of noise cancellation at both $m=1$ and 2 . However, a slight shift in frequency at the first interference peak of $50 \mathrm{~Hz}$ was observed for $2 \mathrm{AH}$. The simple U-shaped cavity $2 \mathrm{AU}$ exhibited the best performance both at $\mathrm{m}=1$ and 2 of 53.55 and $59.23 \mathrm{~dB}$ respectively. Even though at $\mathrm{m}=1$, the waveguides performed closely, at $\mathrm{m}=2$, there was a clear distinction between the waveguides despite the similar $\Delta l$. Overall, towards the high frequencies $2 \mathrm{AU}$ outperformed the other two designs from a TL perspective. This shows that the waveguide tortuosity is critical for HQA when it comes to TL performance and has a substantial impact on acoustic insulation.

The change in tortuosity within a cylindrical dimension of $10 \mathrm{~cm}$ yielded similar TL performance at the first integral frequency, however a change in noise cancellation frequency of $50 \mathrm{~Hz}$ was 
observed. At the second integral frequency $(m=2)$, significant difference in performance was occurred between the tested HQA waveguides. Nevertheless, the waveguide 2AU showed an improvement of $30.25 \%$ in comparison to the solid flat sample followed by $2 \mathrm{AH}$ and $2 \mathrm{AI}$ at $7.25 \%$ and $3.75 \%$ respectively. From the study the tortuosity of the U-shaped HQA waveguide was found to be superior in sound transmission both at $m=1$ and 2 .

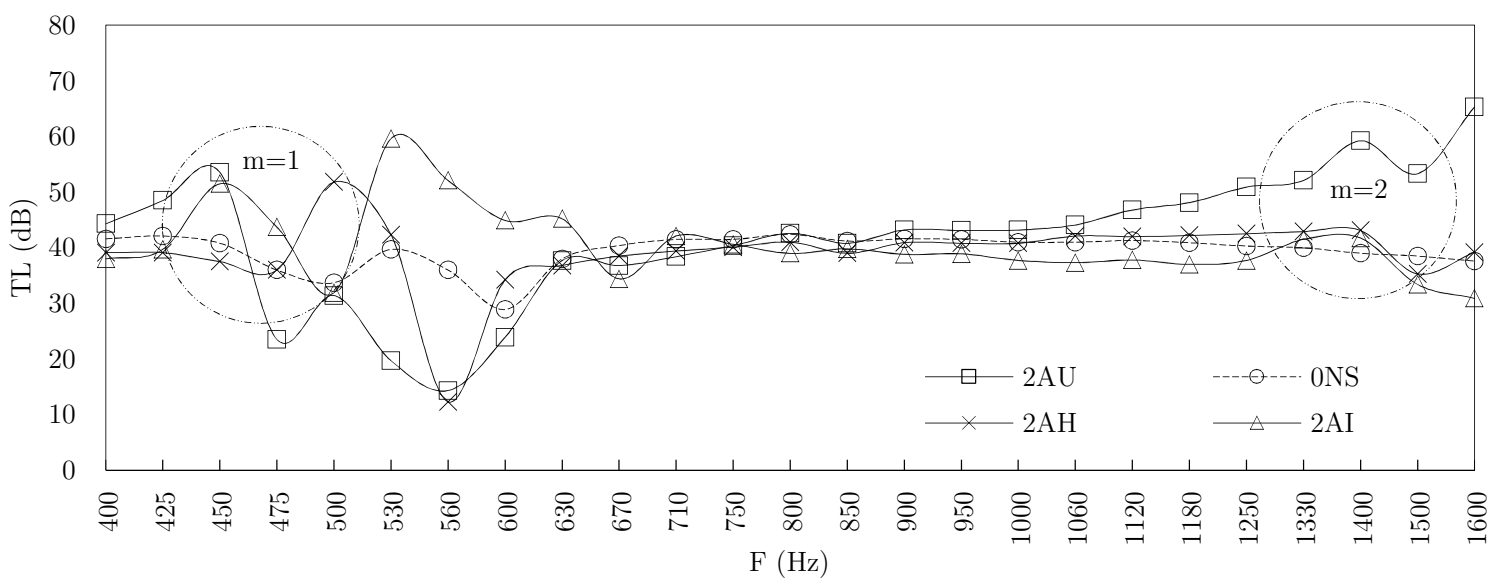

Fig. 19. Influence of waveguide tortuosity on the transmission loss of HQA waveguides.

Overall, the results are encouraging and establishes that HQA waveguides can be used to develop geometry dependent sound absorption and transmission loss that works through passive noise cancellation. The study also demonstrates the $\alpha$ and TL improvements that can be expected from branch-less waveguides and differentiate its performance form quarter-wave resonators. Comparison of the HQA performance with solid flat specimen showed substantial improvement in acoustic insulation despite significant mass and volume reduction. Accordingly, the HQA waveguides using the right parameters associated with $\Delta l, d$ and waveguide tortuosity can deliver significant acoustic performance that allow for frequency customisation.

\section{Conclusions}

Development of HQA waveguides utilising the principles of acoustic wave interference to achieve targeted noise cancellation is demonstrated in this study. The results show that under right geometrical parameters very high sound absorption and transmission loss can be achieved at predetermined frequencies. However, an error $\leq 12 \%$ was found when it comes to the prediction of frequency where peak noise cancellation is expected to happen. When it comes to $\alpha$, the smallest diameter $(d=1.5 \mathrm{~cm})$ outperformed all other designs showing almost complete noise cancellation. However, the TL performance showed an opposite trend with the largest diameter $(d=3 \mathrm{~cm})$ exhibiting improvement of $95.28 \%$ (A) in comparison to a solid flat specimen of same material. Furthermore, the waveguide diameter was found to have an influence of $0.8 f<f<$ $1.2 f$ where the frequency of maximum TL can happen in association with Eqn. (1). Comparison of the HQA waveguide with quarter-wave resonators established that the performance is vastly different and incomparable. Finally, the waveguide tortuosity seems to have a significant impact 
on TL and minimal influence on $\alpha$. Accordingly, this study establishes a new direction in passive acoustic performance that is both material independent and frequency customisable. However, significant further work in terms of numerical modelling and optimisation is required to identify the most optimal HQA waveguide parameters for various applications.

\section{Acknowledgement}

UK Department for Transport funded this research.

\section{References}

[1] Echevarria Sanchez GM, Van Renterghem T, Thomas P, Botteldooren D. The effect of street canyon design on traffic noise exposure along roads. Building and Environment 2016;97:96-110.

[2] Lean G. Noise pollution: why the silence? Virtually no government in the world seems prepared to tackle the problem. The Telegraph 2011.

[3] Arjunan A, Foteinou A. A comparative study on the acoustic behaviour of free-standing curved and flat single panel screens in an open-plan enclosed environment. INTER-NOISE - Int Congr Expo Noise Control Eng : Taming Noise Mov Quiet 2017;2017-January.

[4] Arjunan A, Wang C, English M, Stanford M, Lister P. A Computationally-Efficient Numerical Model to Characterize the Noise Behavior of Metal-Framed Walls. Metals 2015;5:1414.

[5] Arjunan A, Wang CJ, Yahiaoui K, Mynors DJ, Morgan T, Nguyen VB et al. Development of a 3D finite element acoustic model to predict the sound reduction index of stud based double-leaf walls. J Sound Vibrat 2014;333:6140-55.

[6] Arjunan A, Wang CJ, Yahiaoui K, Mynors DJ, Morgan T, English M. Finite element acoustic analysis of a steel stud based double-leaf wall. Build Environ 2013;67:202-10.

[7] Arjunan A, Wang C, Yahiaoui K, Mynors M, Morgan T, Nguyen B et al. Sound frequency dependent mesh modelling to simulate the acoustic insulation of stud based double-leaf walls. Proceedings of the 2014 Leuven Conference on Noise and Vibration Engineering (ISMA2014). 2014.

[8] Hu S, Rajamani R, Yu X. Directional cancellation of acoustic noise for home window applications. Applied Acoustics 2013;74:467-77.

[9] Sas P, Bao C, Augusztinovicz F, Desmet W. Active control of sound transmission through a double panel partition. Journal of Sound and Vibration 1995;180:609-25.

[10] Omoto A, Fujiwara K. A study of an actively controlled noise barrier. J Acoust Soc Am 1993;94:2173-80.

[11] Padhi T, Chandra M, Kar A, Swamy MNS. Design and analysis of an improved hybrid active noise control system. Applied Acoustics 2017;127:260-9.

[12] Sharifzadeh Mirshekarloo M, Tan CY, Yu X, Zhang L, Chen S, Yao K et al. Transparent piezoelectric film speakers for windows with active noise mitigation function. Applied Acoustics 2018;137:90-7.

[13] De Salis MHF, Oldham DJ, Sharples S. Noise control strategies for naturally ventilated buildings. Building and Environment 2002;37:471-84.

[14] Sun Y, Yang T, Zhu M, Pan J. The use of an active controlled enclosure to attenuate sound radiation from a heavy radiator. Journal of Sound and Vibration 2017;392:1-17.

[15] Hasheminejad SM, Keshavarzpour H. Active sound radiation control of a thick piezolaminated smart rectangular plate. Journal of Sound and Vibration 2013;332:4798-816.

[16] M. Guldenschuh, A. Sontacchi, M. Perkmann, M. Opitz. Assessment of active noise cancelling headphones. 2012 IEEE Second International Conference on Consumer Electronics - Berlin (ICCEBerlin) 2012:299-303. 
[17] Huang X, Zou H, Qiu X. A preliminary study on the performance of indoor active noise barriers based on 2D simulations. Build Environ 2015;94, Part 2:891-9.

[18] Chang C, Kuo SM, Huang C. Secondary path modeling for narrowband active noise control systems. Applied Acoustics 2018;131:154-64.

[19] Murao T, Shi C, Gan W, Nishimura M. Mixed-error approach for multi-channel active noise control of open windows. Applied Acoustics 2017;127:305-15.

[20] Desantes JM, Torregrosa AJ, Climent H, Moya D. Acoustic performance of a Herschel-Quincke tube modified with an interconnecting pipe. J Sound Vibrat 2005;284:283-98.

[21] Wang ZH, Hui CK, Ng CF. The acoustic performance of ventilated window with quarter-wave resonators and membrane absorber. Applied Acoustics 2014;78:1-6.

[22] Field CD, Fricke FR. Theory and applications of quarter-wave resonators: A prelude to their use for attenuating noise entering buildings through ventilation openings. Applied Acoustics 1998;53:117-32.

[23] Cai C, Mak CM. Acoustic performance of different Helmholtz resonator array configurations. Applied Acoustics 2018;130:204-9.

[24] Fuller CR, Bies DA. A reactive acoustic attenuator. Journal of Sound and Vibration 1978;56:45-59.

[25] Depollier C, Kergomard J, Lesueur JC. Propagation of low frequency acoustic waves in periodic 2Dlattices of tubes. Journal of Sound and Vibration 1990;142:153-70.

[26] Selamet A, Dickey NS, Novak JM. The Herschel-Quincke tube: A theoretical, computational, and experimental investigation. J Acoust Soc Am 1994;96:3177-85.

[27] Poirier B, Maury C, Ville J. The use of Herschel-Quincke tubes to improve the efficiency of lined ducts. Appl Acoust 2011;72:78-88.

[28] Cai X, Guo Q, Hu G, Yang J. Ultrathin low-frequency sound absorbing panels based on coplanar spiral tubes or coplanar Helmholtz resonators. Appl Phys Lett 2014;105:121901.

[29] Berardi U. Destructive interferences created using additive manufacturing. 2017;45.

[30] Setaki F, Tenpierik M, Turrin M, van Timmeren A. Acoustic absorbers by additive manufacturing. Building and Environment 2014;72:188-200.

[31] Shinsuke N. Sound absorption of Helmholtz resonator included a winding built-in neck extension. INTER-NOISE and NOISE-CON Congress and Conference Proceedings 2016;253:912-9.

[32] Lu Y, Tang H, Tian J, Li H. THE MICRO-SLOTTED RESONATOR WITH FLEXIBLE TUBE BUNDLES. 2007.

[33] Richoux O, Lombard B, Mercier J. Generation of acoustic solitary waves in a lattice of Helmholtz resonators. Wave Motion 2015;56:85-99.

[34] Cambonie T, Mbailassem F, Gourdon E. Bending a quarter wavelength resonator : Curvature effects on sound absorption properties. Applied Acoustics 2018;131:87-102.

[35] Howard CQ, Craig RA. Noise reduction using a quarter wave tube with different orifice geometries. Applied Acoustics 2014;76:180-6.

[36] Greiner S, Wudy K, Lanzl L, Drummer D. Selective laser sintering of polymer blends: Bulk properties and process behavior. Polymer Testing 2017;64:136-44.

[37] Liu Z, Zhan J, Fard M, Davy JL. Acoustic properties of a porous polycarbonate material produced by additive manufacturing. Materials Letters 2016;181:296-9.

[38] Liu Z, Zhan J, Fard M, Davy JL. Acoustic properties of multilayer sound absorbers with a 3D printed micro-perforated panel. Applied Acoustics 2017;121:25-32.

[39] Zhang XH, Qu ZG, He XC, Lu DL. Experimental study on the sound absorption characteristics of continuously graded phononic crystals. AIP Advances 2016;6:105205.

[40] Jiang C, Moreau D, Doolan C. Acoustic Absorption of Porous Materials Produced by Additive Manufacturing with Varying Geometries. 2017.

[41] Benoit B, Camastra C, Kenny M, Li K, Romanowski R, Kevin S. Engineering Silence: Active Noise Cancellation. North Carolina State University 2012. 
[42] Herschel JFW. On the absorption of light by coloured media, viewed in connexion with the undulatory theory. Philos.Mag. 1833;3:401-12.

[43] James M. Fundamental studies of the Herschel-Quincke tube concept with mode measurements. Virginia Tech 2005.

[44] Yongnian Y, Shengjie L, Renji Z, Feng L, Rendong W, Qingping L et al. Rapid Prototyping and Manufacturing Technology: Principle, Representative Technics, Applications, and Development Trends. Tsinghua Science \& Technology 2009.

[45] Bourell DL, Watt TJ, Leigh DK, Fulcher B. Performance Limitations in Polymer Laser Sintering. Physics Procedia 2014;56:147-56.

[46] BSEN ISO 10534-2: 2001. Determination of sound absorption coefficient and impedance in impedance tubes, Part 2: Transfer-function method. 2001.

[47] Arjunan A. Acoustic absorption of passive destructive interference cavities. Materials Today Communications 2019;19:68-75.

[48] BS EN ISO 10140-1: Acoustics. Laboratory Measurement of Sound Insulation of Building Elements. Application Rules for Specific Products. 2014.

[49] Han J, Herrin DW, Seybert AF. Accurate Measurement of Small Absorption Coefficients. SAE International 2007:1-5. 